\title{
Discovery of PAHs in the halo of NGC 5907
}

\author{
J. A. Irwin ${ }^{\star}$ and S. C. Madden
}

Service d'Astrophysique, CEA/Saclay, L'Orme des Merisiers, 91191 Gif-sur-Yvette, France

e-mail: irwin@astro.queensu.ca

Received 13 April 2005 / Accepted 6 September 2005

\section{ABSTRACT}

We have used sensitive archival data from the Infrared Space Observatory (ISO) to make maps of the edge-on low SFR galaxy, NGC 5907, in 6 different MIR bands: $L W 2, L W 5, L W 6, L W 7, L W 8$, and $L W 10$, covering the spectrum from 6.5 to $15.0 \mu \mathrm{m}$ and including several narrow bands that isolate the infrared aromatic spectral features commonly referred to as PAHs. Most of the MIR emission is dominated by PAHs and it is likely that emission from VSGs contribute only negligibly except in the broad IRAS-equivalent band. The flux ratios are typical of galaxies with low SFRs or quiesent regions within galaxies (e.g. M 83) and a very high PAH/continuum ratio is observed. The PAH emission follows the $\mathrm{CO}$ distribution and also shows some correlation within the disk with the $\lambda 850 \mu \mathrm{m}$ distribution. However, the PAH emission also reaches larger galactocentric radii than the $\mathrm{CO}$ and other correlations suggest that the PAHs are also more widespread. A significant new discovery is the presence of PAHs in the halo of the galaxy. In the narrow bands that isolate single PAH features, the emission shows structure similar to high latitude features seen in other galaxies in other tracers. The features extend as far as $6.5 \mathrm{kpc}$ from the plane but scale heights of $3.5 \mathrm{kpc}$ are more typical. The $\lambda 11.3 / \lambda 7.7$ ratio also appears to increase with distance from the major axis. To our knowledge, this is the first time PAHs have been seen in the halo of an external galaxy. Just as significantly, they are seen in a low SFR galaxy, suggesting that strong SNe and winds are not necessary for these large molecules to reach high latitudes.

Key words. galaxies: individual: NGC 5907 - galaxies: halos - galaxies: ISM

\section{Introduction}

The galactic disk-halo interface is a critical region connecting two very different environments over a relatively small vertical distance. The prevalence, nature, and underlying physical drivers of features which span this region and extend into the halo are particularly important since they play a role in the energy balance as well as the chemical evolution of a galaxy. Moreover, knowledge of the physical conditions of outflowing gas in nearby galaxies can provide important constraints on galaxy formation, since outflows (i.e. some form of "feedback") are crucial to galaxy formation scenarios (e.g. Dekel \& Silk 1986; Navarro \& White 1993; Mo \& Mao 2004; Scannapieco et al. 2002; Kay et al. 2002; Zhao et al. 2003a,b; Sommer-Larsen et al. 2003; Monaco 2004; Marri \& White 2003). Nearby edge-on galaxies therefore, provide an important local laboratory within which such flows can be examined in some detail.

Evidence for outflows includes optical emission line cones from nuclear starbursts (e.g. Ohyama et al. 2002; Veilleux et al. 2003, and others), $\mathrm{H} \alpha$ disk-halo filaments (Rossa \& Dettmar 2003), X-ray halos (Wang, Chaves, \& Irwin 2003; Strickland et al. 2004a,b), and supershells/bubbles in HI (e.g. Spekkens et al. 2004). Dust in halos has been observed in

* On leave from the Dept. of Physics, Engineering Physics \& Astronomy, Queen's University, Kingston, K7L 3N6, Canada. absorption against the background stellar continuum (Sofue 1987; Ichikawa et al. 1994; Alton et al. 1999; Howk \& Savage 1999; Thompson et al. 2004), although this only probes halo dust fairly close to the disk. More recently, however, IR, mm, and sub-mm emission from dust has also been detected in halos (Alton et al. 1998; Neininger \& Dumke 1999; Brar et al. 2003; Popescu et al. 2004; Tuffs \& Popescu 2005). The physical conditions in such halos are, however, not well understood, especially those which allow the survival of dust in hot rarefied halo environments. Indeed, the conditions of dust survival in halos may be similar to those of elliptical galaxies (Tsai \& Mathews 1995; Xilouris et al. 2004).

The mid-infrared (MIR) wavelength regime has the potential to characterise the dust properties in halos. The sensitive ISOCAM detector (Cesarsky et al. 1996) on board the Infrared Space Observatory (ISO) satellite (Kessler et al. 1996) provides necessary spatial resolution and, with an appropriate selection from the vast assortment of filters (from 4 to $18 \mu \mathrm{m}$ ), it is possible to discriminate between contributing sources of dust. By now, almost a decade after the launch of ISO, we are beginning to understand the properties of the various components in the MIR regime and what they are tracing in galaxies. ISOCAM surveys of galaxies (Rigopoulou et al. 1999; Dale et al 2000; Laurent et al. 2000; Roussel et al. 2001a; Sturm et al. 2002) and detailed studies of individual galaxies (e.g. Galliano et al. 2003; Vogler et al. 2005) have brought deep insight into the 
extragalactic MIR picture. Among the most prominent of the MIR features in galaxies are the carriers of the unidentified infrared bands, one model for these being the PAHs (polycyclic aromatic hydrocarbons) ${ }^{1}$ and small hot (typically a few hundred K, see Sect. 3) grains.

In this study, we use the very sensitive previously unpublished observations of NGC 5907 in the ISO database. This has allowed us to take advantage of an extraordinary number of filters that were used to observe both along the disk as well as into the halo region, where we make clear detections far from the plane. Combining these data, we are able to delve into the MIR properties of the halo. This paper is organized as follows. In Sect. 1.1 we review the previous observations of NGC 5907, in Sect. 2 we discuss the observations and data reduction, Sect. 3 reviews the various contributors to the MIR observing bands, Sect. 4 presents the results for both the disk and halo regions, Sect. 5 provides the discussion and the conclusions are given in Sect. 6.

\subsection{NGC 5907}

NGC 5907 (Table 1), at a distance of $11 \mathrm{Mpc}(1 \operatorname{arcsec}=$ $53.3 \mathrm{pc}$ ), is one of the largest edge-on systems in the sky. Its nucleus is an HII region type and no nuclear radio source has been reported (Ulvestad \& Ho 2002) in spite of some efforts to find one. Non-circular molecular gas motions near the nucleus have been interpreted in terms of a bar (Garcia-Burillo et al. 1997; Garcia-Burillo \& Guelin 1997); however, there is no evidence for a bar in the near infra-red (Jarrett et al. 2003). The CO distribution is centrally peaked (Dumke et al. 1997) with a distribution suggestive of rings or spiral arms (Sofue 1994; Dumke et al. 1997).

NGC 5907 has been an important target in searches for faint stellar halos since it has no appreciable bulge and was originally thought to be isolated. Sackett et al. (1994) first reported the detection of an $R$-band halo to a distance of $6 \mathrm{kpc}$ around this galaxy, following which $V$ and $I$ band extraplanar light was detected by Lequeux et al. (1996), as well as $J$ and $K$ band halos by James \& Casali (1998) and Rudy et al. (1997).

NGC 5907 is, in fact, a member of the 396th Lyon Group of Galaxies (LGG 396) of which there are 4 identified members: NGC 5866, NGC 5879, NGC 5907, and UGC 9776 (Garcia 1993). These members are at large separations (Table 2) and are unlikely to be interacting with NGC 5907 now. However, the more recent detection of a nearby companion, PGC 54419, at a projected distance of only $36.9 \mathrm{kpc}$ and velocity separation of $\Delta V=45 \mathrm{~km} \mathrm{~s}^{-1}$ (Shang et al. 1998) indicate that NGC 5907 is not as isolated as previously thought. Moreover, their additional discovery of a large optical ring around the galaxy indicates that some interaction has indeed occurred. A pronounced HI warp (Sancisi 1976) and some evidence for an optical warp (Morrison et al. 1999) are consistent with this. The ring and nearby companion are faint in comparison to NGC 5907; the luminosity of the ring is $\leq 1.2 \%$ of NGC 5907 and the mass

\footnotetext{
1 The carriers of the MIR bands are not certain, but in this paper, we adopt the PAH nomenclature for discussion and comparison with other authors.
}

of the second companion is $0.5 \%$ of the mass of NGC 5907 (Shang et al. 1998). Dynamical modelling of the ring indicates that it was formed at least $0.8 \mathrm{Gyr}$ ago via the destruction of a satellite of mass $2 \times 10^{8} M_{\odot}$ (Johnston et al. 2001, who adopted a slightly larger distance to the galaxy). Follow-up observations showed that the ring is highly asymmetric and suggests that NGC 5907 does not after all have a faint extended stellar halo as originally envisioned (Zheng et al. 1999). A nearinfrared (3.5-5 $\mu \mathrm{m})$ search also failed to show evidence for a halo associated with NGC 5907 (Yost et al. 1999) and HST observations find fewer bright giants in the halo region than would be expected from a halo with standard dwarf-to-giant ratios (Zepf et al. 2000). Recent 2MASS $K_{\mathrm{s}}$ band observations of NGC 5907 (Bizyaev \& Mitronova 2002) provide a stellar scale height of $z_{0}=0.49 \mathrm{kpc}$ with an isothermal assumption [i.e. $\left.\rho(z) \propto \sec ^{2}\left(z / z_{0}\right)\right]$.

As for a gaseous halo, no high latitude ionized gas has yet been detected (Rand 1996) nor did Howk \& Savage (1999) detect any extraplanar dust in optical absorption. Dumke et al. (1997) find a CO FWHM of 400 pc and an HI FWHM of $1.5 \mathrm{kpc}$, although the warp makes this latter value uncertain. The most extended component appears to be the radio continuum (Dumke et al. 2000), for which an exponential thick disk component is found with a scale height of $1.5 \mathrm{kpc}$. An $850 \mu \mathrm{m}$ map has recently been published by Alton et al. (2004) who find an extraplanar exponential scale height of $0.11 \mathrm{kpc}$.

ISO 60,100 , and $180 \mu \mathrm{m}$ fluxes as well as a $12 \mu \mathrm{m}$ image have been published by Bendo et al. (2002), and Bendo et al. (2003) also present temperature fits to the data. More recently, Alton et al. (2004) have also fit a two-component dust model to NGC 5907 which includes newer $850 \mu \mathrm{m}$ data from observations using the Submillimetre Common Use Bolometric Array (SCUBA) on the James Clerk Maxwell Telescope. These temperatures are presented in Table 1. An earlier 2-component dust temperature model of $18 \mathrm{~K}$ and $54 \mathrm{~K}$ had been reported by Dumke et al. (1997) who observed the $1.2 \mathrm{~mm}$ dust component. Note that two-temperature models are simply a rough approximation to the range of temperatures that likely exist in this galaxy. Other parameters for NGC 5907 are also listed in Table 1 including the star formation rate $(S F R)$ which is quite low (cf. $S F R=3.8 M_{\odot} \mathrm{yr}^{-1}$ for the quiescent galaxy, NGC 891, Popescu et al. 2000). In Table 1 we also provide comparative data on the nearby galaxy, M 83, whose spectral energy distribution (SED) has recently been studied in some detail (Vogler et al. 2005) and with which we compare our NGC 5907 results.

\section{Observations and data reduction}

\subsection{Observations}

Data on NGC 5907 were available in filters, denoted $L W n$, where $L W$ indicates the long wavelength array of the ISOCAM detector and $n$ specifies the wavelength band. Details of the data acquisition and resulting maps are provided in Table 3. As we prefer to designate the bands according to their wavelength, we use the following nomenclature: $L W 5, L W 6, L W 7, L W 8$, $L W 2$ and $L W 10$ are referred to as $6.8 \mathrm{~N}, 7.7 \mathrm{~N}, 9.6 \mathrm{~N}, 11.3 \mathrm{~N}$, $6.7 \mathrm{~W}$, and $12 \mathrm{~W}$, respectively, where the number represents 
Table 1. Basic galaxy parameters.

\begin{tabular}{|c|c|c|}
\hline Parameter & NGC 5907 & $\overline{\mathrm{M} 83}$ \\
\hline $\mathrm{RA}(\mathrm{J} 2000)^{a}(\mathrm{~h} \mathrm{~m} \mathrm{~s})$ & 151553.69 & \\
\hline $\operatorname{Dec}(\mathrm{J} 2000)^{a}\left({ }^{\circ}, \prime \prime\right)$ & 561943.9 & \\
\hline$V_{\text {hel }}^{a}\left(\mathrm{~km} \mathrm{~s}^{-1}\right)$ & 667 & \\
\hline Distance (Mpc) & $11^{b}$ & $4.5^{c}$ \\
\hline Morph. type $^{a}$ & SA(s)c: sp HII: & $\mathrm{SAB}(\mathrm{s}) \mathrm{c} ; \mathrm{HII}$ \\
\hline Incl. $\left({ }^{\circ}\right)$ & $86.5^{d}$ & $27^{e}$ \\
\hline Major $\times$ Minor Axis Diameters $^{a}\left({ }^{\prime} \times{ }^{\prime}\right)$ & $12.77 \times 1.40$ & $12.9 \times 11.5$ \\
\hline Major Axis Diameter (kpc) & 41 & 17 \\
\hline$f_{60} / f_{100}^{f}$ & 0.24 & 0.54 \\
\hline$L_{I R}^{f}\left(L_{\odot}\right)$ & $7.1 \times 10^{9}$ & $1.4 \times 10^{10}$ \\
\hline$T_{\text {dust }^{g}} 1-\operatorname{Comp}\left(\lambda^{-1}\right)$ & $26 \mathrm{~K}$ & 36 \\
\hline$T_{\text {dust }^{g}}{ }^{g}$-Comp $\left(\lambda^{-2}\right)$ & $22 \mathrm{~K}$ & 29 \\
\hline$T_{\text {dust }^{g}} 2$-Comp $\left(\lambda^{-2}\right)$ & $13,26 \mathrm{~K}$ & \\
\hline$T_{\text {dust }}{ }^{g} 2-\operatorname{Comp}\left(\lambda^{-1.5}\right)$ & $13,28 \mathrm{~K}$ & \\
\hline$M_{\text {dust }}\left(M_{\odot}\right)$ & $6.0 \times 10^{7 h}, 3.2 \times 10^{7 i}$ & $1.8 \times 10^{6 i}$ \\
\hline$\Sigma_{\text {dust }^{j}}^{j}\left(M_{\odot} \mathrm{kpc}^{-2}\right)$ & $7.9 \times 10^{5}$ & $2.6 \times 10^{5}$ \\
\hline $\operatorname{SFR}\left(M_{\odot} \mathrm{yr}^{-1}\right)$ & $2.2^{h}, 1.2^{k}$ & $2.4^{j}$ \\
\hline$S F R / \mathrm{A}^{l}\left(M_{\odot} \mathrm{yr}^{-1} \mathrm{kpc}^{-2}\right)$ & $1.7 \times 10^{-3}, 9.0 \times 10^{-4}$ & $1.1 \times 10^{-2}$ \\
\hline
\end{tabular}

a From the NASA Extragalactic Database (NED). This center is the 2MASS center which agrees with the dynamical center (Dumke et al. 1997) to within $\triangle \mathrm{RA}=0.8^{\prime \prime}$ and $\Delta \mathrm{Dec}=0.3^{\prime \prime}$.

$b$ Sasaki (1987).

c Thim et al. (2003).

${ }^{d}$ Garcia-Burillo et al. (1997).

$e$ From the major and minor axis diameters and a thin disk assumption.

$f$ Flux ratio or FIR luminosity, using the 60 and $100 \mu \mathrm{m}$ IRAS fluxes from Sanders et al. 2003. $L_{\mathrm{IR}}$ allows for extrapolations below 40 and above $120 \mu \mathrm{m}$ and adjusts to the distance given in this table.

$g$ Dust temperature, assuming the emissivity laws shown, for one or two component fits (Bendo et al. 2003). For M 83, a 2-component model does not improve the fit. The second line for the 2-component model gives the fit from Alton et al. (2004).

$h$ From Misiriotis et al. (2001).

${ }^{i}$ From Bendo et al. (2003) for fluxes within a 135" aperture, assuming a $\lambda^{-2}$ emissivity and adjusting to the distances in this table.

${ }^{j}$ Dust surface density, using the dust masses in this table (from the adjusted Bendo et al. data) and their aperture sizes.

${ }^{k}$ From $L_{\mathrm{IR}}$ of this table and the calibration of Kennicutt (1998).

$l$ SFR per unit area, from values in the immediately preceding row and the optical major axes given in this table.

Table 2. Galaxy group membership ${ }^{a}$.

\begin{tabular}{lccccc}
\hline \hline Galaxy Name & $\begin{array}{c}\text { Velocity } \\
\left(\mathrm{km} \mathrm{s}^{-1}\right)\end{array}$ & $\begin{array}{c}\text { RA } \\
(\mathrm{h} \mathrm{m} \mathrm{s})\end{array}$ & $\begin{array}{c}\text { Dec } \\
\left({ }^{\circ} \prime^{\prime \prime}\right)\end{array}$ & $\begin{array}{c}\text { Separation }^{b} \\
(\text { Arcmin })\end{array}$ & $\begin{array}{c}\text { Separation }^{b} \\
(\text { Radii })\end{array}$ \\
\hline NGC 5907 & 667 & 151553.69 & 561943.9 & - & \\
NGC 5866 & 672 & 150629.56 & 554547.9 & 85.8 & 13.4 \\
NGC 5879 & 772 & 150946.77 & 570000.7 & 64.5 & 10.1 \\
UGC 9776 & 833 & 151307.44 & 565807.3 & 44.7 & 7.0 \\
PGC 54419 & 712 & 151448.04 & 562715.4 & 11.8 & 1.8 \\
\hline
\end{tabular}

a Members of the Galaxy Group LGG 396 (Garcia 1993) as well as PGC companion found by Shang et al. (1998). All values are taken from NED.

$b$ "Separation" indicates the distance of the group member from the center of NGC 5907 in units of arcmin and in units of the radius of NGC 5907 (see Table 1).

the central wavelength of the filter and N/W refers to a "narrow"/"wide" band.

The first 4 data sets were taken on 23 Feb. 1996 in standard raster mode (CAM01) in the narrow bands, $6.8 \mathrm{~N}, 7.7 \mathrm{~N}$, $9.6 \mathrm{~N}$, and $11.3 \mathrm{~N}$. Each observation had 3 different on-source pointings for a total field which provided full coverage of the south-east part of the galaxy as well as sufficient coverage far from the emission for sky subtraction. For each pointing, a series of frames were taken over a period of time in a "temporal block". The approximate number and duration of the frames are given in Table 3.

The 5th data set was taken on 12 Aug. 1996 (12W). These data were taken in microscan raster mode (CAM01) with 12 observing positions in a $6 \times 2$ pattern (Bendo et al. 2002) 
Table 3. Observing and map parameters.

\begin{tabular}{|c|c|c|c|c|c|c|}
\hline Parameter & $6.8 \mathrm{~N}(L W 5)$ & $7.7 \mathrm{~N}(L W 6)$ & $9.6 \mathrm{~N}(L W 7)$ & $11.3 \mathrm{~N}(L W 8)$ & $12 \mathrm{~W}(L W 10)$ & $6.7 \mathrm{~W}(L W 2)$ \\
\hline Central Wavelength $^{a}(\mu \mathrm{m})$ & 6.8 & 7.7 & 9.6 & 11.3 & 12.0 & 6.7 \\
\hline Frequency Range $^{a}(\mu \mathrm{m})$ & $6.5-7.0$ & $7.0-8.5$ & $8.5-10.7$ & $10.7-12.0$ & $8.0-15.0$ & $5.0-8.5$ \\
\hline Observing Mode ${ }^{a b}$ & CAM01 & CAM01 & CAM01 & CAM01 & CAM01 & CAM03 \\
\hline \multirow[t]{4}{*}{ TDT No. ${ }^{b}$} & 09801401 & 09801401 & 09801401 & 09801401 & 26902911 & 15601301 \\
\hline & & & & & & 15601202 \\
\hline & & & & & & 15601103 \\
\hline & & & & & & 15601004 \\
\hline Pixel Field of View (arcsec) & 6.0 & 6.0 & 6.0 & 6.0 & 1.0 & 6.0 \\
\hline $\operatorname{PSF}(F W H M)(\operatorname{arcsec})$ & 7.2 & 7.2 & 7.2 & 7.2 & 4.2 & 7.2 \\
\hline Date of Observations & 23 Feb. 96 & 23 Feb. 96 & 23 Feb. 96 & 23 Feb. 96 & 12 Aug. 96 & 21 Apr. 96 \\
\hline No. of On-Source Pointings ${ }^{c}$ & 3 & 3 & 3 & 3 & 12 & 4 \\
\hline Mean No. Frames per Pointing & 16 & 16 & 17 & 17 & 15 & 248 \\
\hline Integration Time per Frame (s) & 10.0804 & 10.0804 & 10.0804 & 10.0804 & 1.12 & 10.0804 \\
\hline Total On-Source Time ${ }^{d}(\mathrm{~min})$ & 8.1 & 8.1 & 8.6 & 8.6 & 3.4 & 167 \\
\hline Sky Coverage $^{e}\left(\operatorname{arcmin}^{2}\right)$ & $8.2 \times 3.2$ & $8.2 \times 3.2$ & $8.2 \times 3.2$ & $8.2 \times 3.2$ & $2.8 \times 1.6$ & 8.4 \\
\hline Calibration Error $^{a}(\%)$ & 7.5 & 6.4 & 6.7 & 5.4 & 3.9 & 3.3 \\
\hline Sky Level $^{f}\left(\right.$ mJy pixel $\left.^{-1}\right)$ & 1.39 & 3.28 & 7.19 & 10.27 & 0.27 & 1.99 \\
\hline $1 \sigma$ Sky Error $^{g}\left(\mathrm{mJy}\right.$ pixel $\left.^{-1}\right)$ & 0.24 & 0.15 & 0.28 & 0.30 & 0.015 & \\
\hline$\sigma_{\mathrm{rms}}^{h}\left(\mathrm{mJy}\right.$ pixel $\left.^{-1}\right)$ & 0.44 & 0.17 & 0.15 & 0.24 & 0.062 & \\
\hline Transient $\mathrm{Rms}^{i}\left(\mathrm{mJy} \mathrm{pixel}^{-1}\right)$ & 0.35 & 0.24 & 0.16 & 0.23 & 0.044 & \\
\hline$\sigma^{j}\left(\mathrm{mJy}\right.$ pixel $\left.^{-1}\right)$ & 0.61 & 0.33 & 0.36 & 0.45 & 0.077 & $0.025^{k}$ \\
\hline$\sigma^{l}\left(\mathrm{mJy} \operatorname{arcsec}^{-2}\right)$ & 0.017 & 0.0092 & 0.010 & 0.013 & 0.077 & 0.00069 \\
\hline Dynamic Range $^{m}$ & 20 & 72 & 28 & 41 & 6.9 & 702 \\
\hline
\end{tabular}

a Blommaert et al. (2003).

${ }^{b}$ CAM01 is the general observing mode for photometric imaging. CAM03 is a beam-switching mode for photometric imaging. TDT No. is a unique number that identifies the observation.

$c$ This is the number of different pointing positions on-source; for some pointings, the fields overlap.

$d$ Total integration time per on-source pointing times the number of pointings. Time on sky not included.

$e$ Total area of sky covered after registration of the different on-source pointings.

$f$ Median value of the histogram of all sky pixel values which were subtracted from the image.

$g 1 \sigma$ width of the histogram of sky pixel values which were used to determine the sky background median.

${ }^{h}$ Median $\sigma_{\text {rms }}$ over the field, where the $\sigma_{\text {rms }}$ is the per pixel error from the dispersion in the signal carried through by the software throughout the data reductions.

${ }^{i}$ Median rms over the field, where the rms is the error due to transients.

${ }^{j}$ Quadratic sum of the random errors from the immediately preceding 3 rows, unless otherwise indicated. This error represents a typical random error for any pixel in the map, but there are positions at which the error is larger or smaller. Note that the calibration error is not included.

${ }^{k}$ Median rms error over the 4 fields which constitute the mosaic, including all applicable random errors. For this beam-switching observation, the above errors were factored into this error in the software and were not calculated separately.

${ }^{l}$ Random error from previous row per 1 arcsec square pixel.

$m$ Maximum of map divided by the median Random error.

with a resulting $1^{\prime \prime}$ pixel spacing. In this case, 12 on-source pointings were made for full coverage of the central part of the galaxy with sufficient sky coverage for subtraction.

The final data set, 6.7W, was obtained on 21 Apr. 1996 in beam switching mode (CAM03). There were 4 positions on-source: one containing the galaxy center but offset to the south-west, another containing the galaxy center offset to the north-east, one at the end of the major axis to the north and one at the end of the major axis at the south. Thus, the final combined image does not provide full coverage of the whole galaxy but rather samples the galaxy at four locations. For each of the 4 pointings, the off-source sky positions were placed at a variety of position angles with respect to that pointing.

\subsection{Reductions}

All data were reduced using the CAM Interactive Reduction Package (CIR, Chanial 2003). First, the dark current was subtracted following the method of Biviano et al. (1998) which includes a dark correction, a second-order dark correction depending on detector temperature and time of observation, and a short-drift correction. Next, high glitches due to cosmic ray (CR) hits were removed automatically via a multi-resolution filtering technique (Starck et al. 1999) at a $6 \sigma$ level. The effects of memory on a pixel (transient effects) were then corrected (de-glitched) using the Fouks-Schubert method (Coulais \& Abergel 2000). Each data set was then examined carefully, frame by frame, and further bad pixels were removed manually 


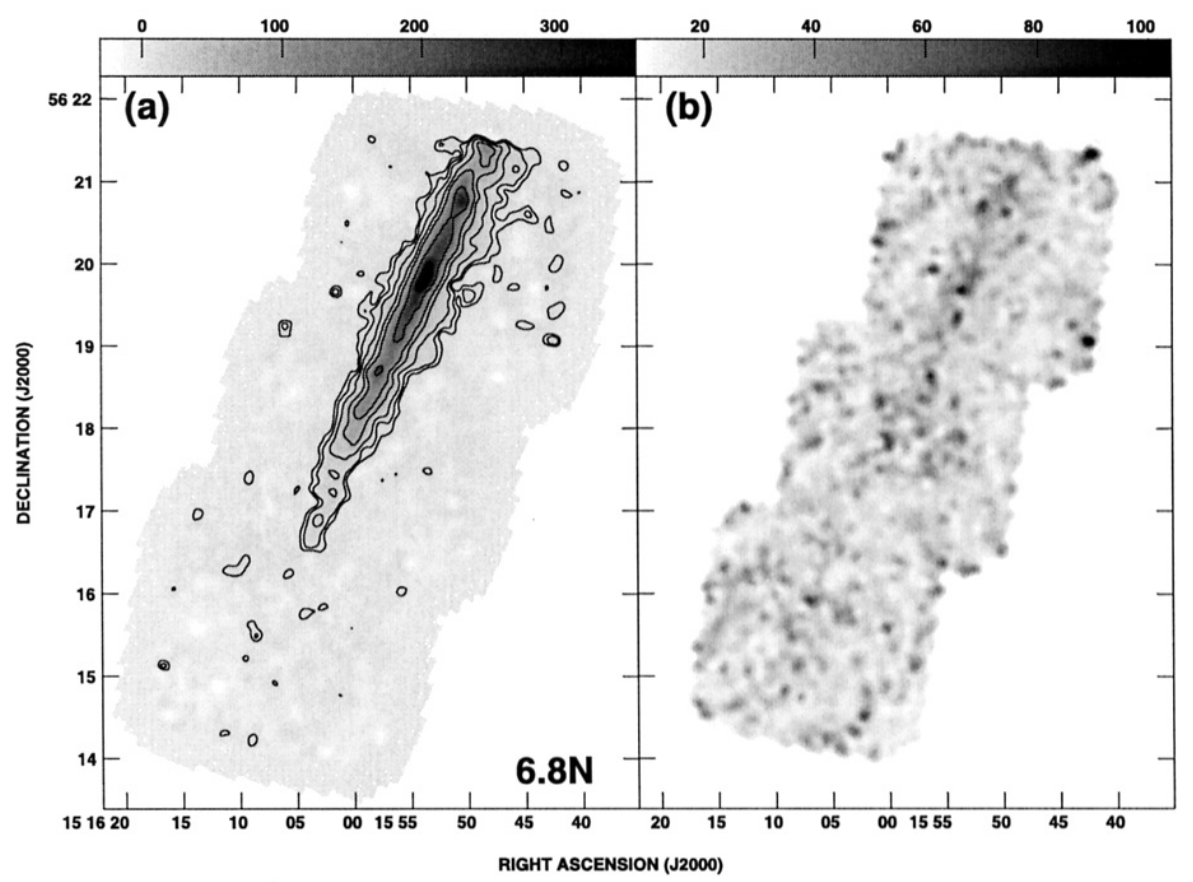

Fig. 1. Maps of NGC 5907 in the $6.8 \mathrm{~N}$ band showing a) total emission and b) the Random error map (see Sect. 2). The greyscale is in units of $\mu \mathrm{Jy} \operatorname{arcsec}^{-2}$ and ranges from the minimum to maximum map values. Contours are at 0.012 $\left(2 \sigma_{\text {map }}\right), 0.018,0.03,0.06,0.10,0.20$, and $0.30 \mathrm{mJy} \operatorname{arcsec}^{-2}$ where $\sigma_{\text {map }}$ is the rms noise level as measured from the emission map itself. as required. Most of these occurred in regions immediately adjacent to pixels which had been automatically de-glitched.

For the raster mode observations (CAM01: 6.8N, 7.7N, $9.6 \mathrm{~N}, 11.3 \mathrm{~N}$, and $12 \mathrm{~W})$, the edges were then trimmed (blanked), the frames were corrected for jitter, and averaged. The results at each pointing were then corrected for the flat field according to the on-line library of calibration flat fields (see Roussel et al. 2001a). The various pointings on-source were then projected onto a common grid in sky coordinates and corrected for field lens distortion. At this point, the data were calibrated to $\mathrm{mJy} / \mathrm{pixel}$ units (Blommaert et al. 2003). Finally, the sky background/foreground was subtracted by first isolating a region of sky-only in the field and subtracting the median of that region from the field.

For the beam switching observations (CAM03: 6.7W) we carried out an additional stage of manual deglitching to account for the fact that in the off-source positions, the CCD contained a fading memory of the galaxy itself from when the array pointed at the galaxy. Thus, the region of the array which had previously contained the galaxy was blanked in approximately 10 sky frames immediately after moving from the galaxy to the sky. This blanking covered about $20 \%$ of the array and left approximately 20 frames where the image had faded and blanking was no longer required. This additional deglitching was required only for the center-most two fields, and not for the two fields at the ends of the galaxy's major axis, given the weakness of the signal there. Beam switching allowed for sufficient sky coverage off source that the sky positions provided both the flat field and its distortions as well as the sky brightness values. Thus, after the average on-source image was created, it was corrected using an image that was formed from an average of the various off-source positions. The result was then calibrated to $\mathrm{mJy} /$ pixel units. This mode of operation provides the best sky subtraction because it does this pixel by pixel rather than using a single value (the median of the sky values) for all pixels, as above. Finally the registration and combination of the 4 pointings, as well as an interpolation over one bad column on the array was carried out outside of the CIR package. In the region of the galaxy in which there was overlap (see center region of Fig. 6a), the flux levels between the two overlapping fields differed by $\sim 4.5 \%$.

All images were interpolated/rotated into RA/Dec coordinates with 1 arcsec square pixels for consistency. The resulting maps are shown in Fig. 1a through 6a. The lowest contour, in each case, is set to $2 \sigma_{\text {map }}$, where $\sigma_{\text {map }}$ is the rms variation calculated from a blank sky region on the emission map itself. The field of view is accurately shown by the error maps (next section). The results are discussed in Sect. 4.

\subsection{Error analysis}

Attention has been paid to the error budget in the final images. The resultant error is the quadratic sum of individual errors, consisting of a) the readout and photon noises added quadratically and calculated by the software, denoted " $\sigma_{\text {rms }}$ " in Table 3 , b) an error on the background level which was subtracted; this is taken as the $1 \sigma$ dispersion in the histogram of values used to determine the sky value to be subtracted and includes uncertainties due to residual dark current, flat field, glitches, remnant images, and long-term drifts, denoted " $1 \sigma$ Sky Error" in Table 3, and c) the effects of the non-stabilization of the signal (memory) as a function of time over a temporal frame-byframe block at any pointing; this error has been estimated from variations in the signal that are greater than $3 \sigma$ within a block (assuming that such larger variations are caused by only this error) and applying a factor which accounts for the fact that the error is worse near the beginning of a block (see Galliano 2004; Roussel et al. 2001a), denoted "Transient rms" in Table 3. The quadratic sum of these errors is referred to as the random error (" $\sigma$ " in the table) and has been computed "per pixel", 

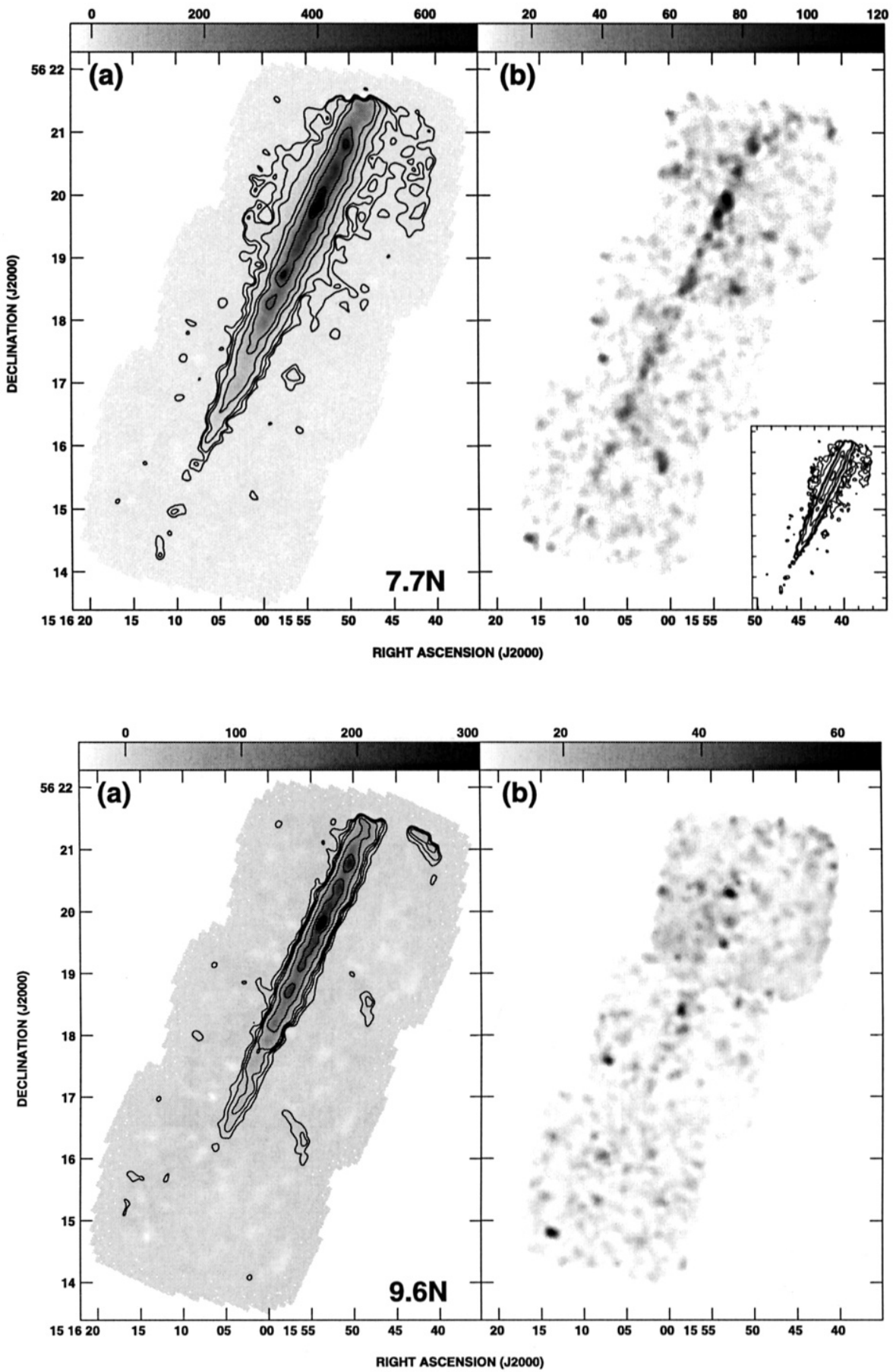

Fig. 2. As in Fig. 1, but for $7.7 \mathrm{~N}$ and with contours at $0.006\left(2 \sigma_{\text {map }}\right), 0.01,0.018,0.05$, $0.10,0.300 .50$, and $0.65 \mathrm{mJy} \operatorname{arcsec}^{-2}$. The inset shows the emission after an estimate for the stellar contribution has been subtracted (see Sect. 3.1).

Fig. 3. As in Fig. 1, but for $9.6 \mathrm{~N}$ with contours at $0.015\left(2 \sigma_{\text {map }}\right), 0.025,0.035,0.05$, $0.10,0.18$, and $0.25 \mathrm{mJy} \operatorname{arcsec}^{-2}$. where the pixel is the originally sized pixel given in the table, and also has been computed "per square arcsec", after interpolating onto a 1 arcsec pixel grid. We formed a total Random Error map (showing $\sigma_{i}$ for each map point, i, from the quadratic sum of the $\sigma_{\text {rms }}$ map, the Transient rms map, plus a constant $1 \sigma$ Sky Error value. These maps are shown in Figs. $1 \mathrm{~b}$ through 5b. However, for the beam-switching observations, since we also have the sky background errors for each pixel (rather than the same sky value for every pixel), all three sources of error were calculated in one step (thus the missing intermediate step values in Table 3) and the final Random Error map is shown in Fig. 6b.
The calibration error (see Table 3 ) has not been included in Figs. $1 \mathrm{~b}$ through $5 \mathrm{~b}$ since this would globally raise or lower the flux level of the map and these maps are mainly meant to show variations across the fields. However, it is included in any calculations of flux density. A remaining error, that of variations in the flux density calibration according to orbit $(\approx 5 \%$, Blommaert et al. 2003) has not been included. Since $6.8 \mathrm{~N}, 7.7 \mathrm{~N}, 9.6 \mathrm{~N}$, and $11.3 \mathrm{~N}$ observations were all taken at the same orbital point, this error does not enter into a field by field comparison of these 4 bands. However, it will be a factor in a comparison between these bands and the remaining two. 

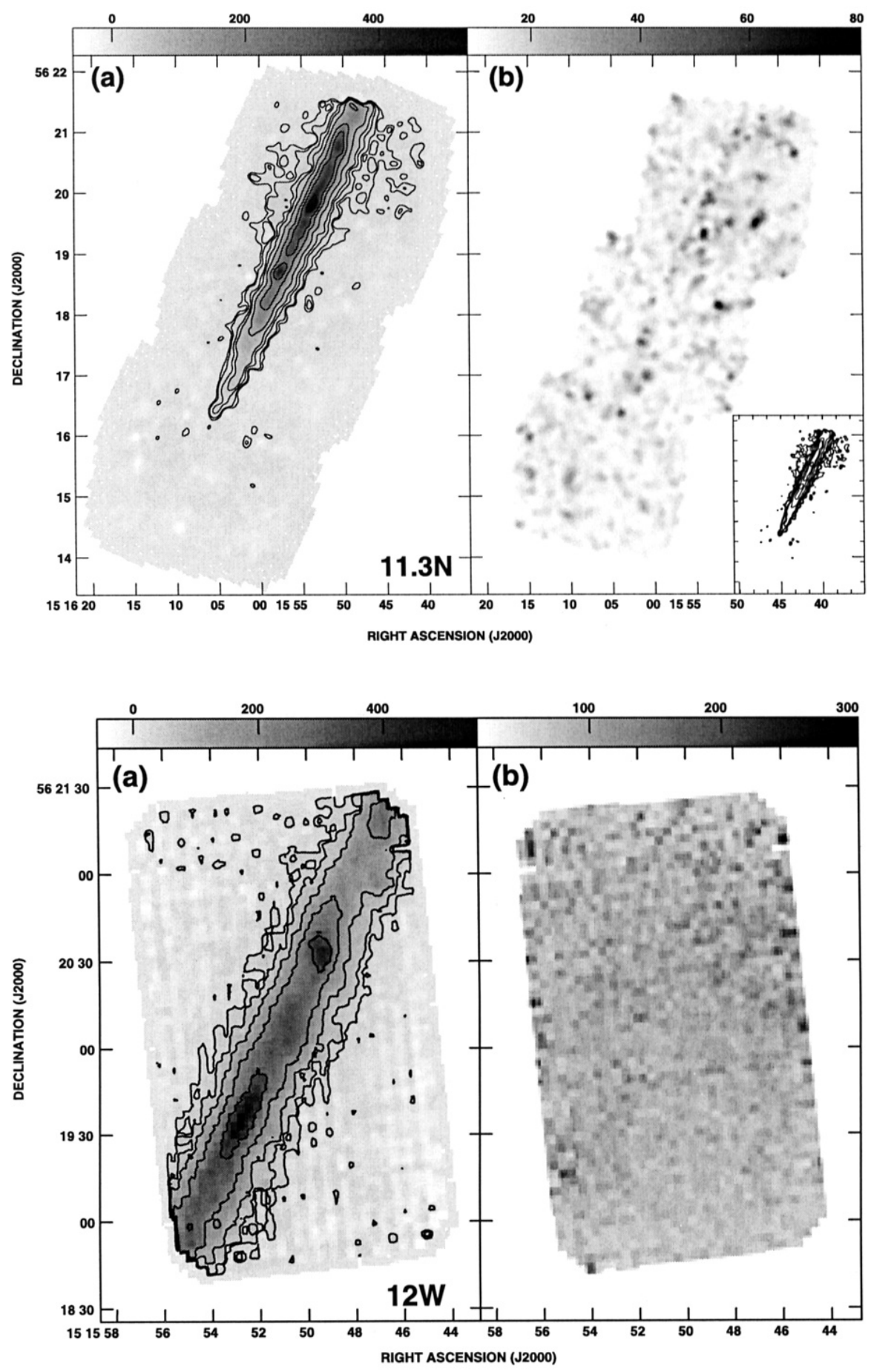

Fig. 4. As in Fig. 2, but for $11.3 \mathrm{~N}$ with contours at $0.016\left(2 \sigma_{\text {map }}\right), 0.022,0.038,0.055$, $0.10,0.20,0.30$, and $0.45 \mathrm{mJy} \operatorname{arcsec}^{-2}$.
Fig. 5. As in Fig. 1, but for $12 \mathrm{~W}$ with contours at $0.028\left(2 \sigma_{\text {map }}\right), 0.05,0.10,0.20$, and $0.35 \mathrm{mJy} \operatorname{arcsec}^{-2}$.
We note that the true errors cannot be determined rigorously, for example, the memory effects of the camera are not accurately known (Roussel et al. 2001a), although they can be tested for, as described below. Thus the quoted errors, though calculated rigorously, should be considered indicative. An example is seen in Fig. $3 \mathrm{a}$ in which there remain three residual artifacts to the west of the galaxy which correspond to the edges of the three fields. We have also, therefore, shown the lowest contour level in Figs. 1a through 6 a as twice the rms level on the emission map itself, the latter being an indication of the pixel-to-pixel variation across a field in the region of the sky.
Since we are reporting the discovery of halo PAHs in NGC 5907 in bands, 7.7N, 11.3N, and 6.7W (see Sect. 4.2), we performed some additional tests which were designed to check whether this high latitude emission might have been produced artificially. These tests are as follows, foccusing on the $7.7 \mathrm{~N}$ band.

We first examined the point spread function (PSF) whose $F W H M$ values are 7.2" in these bands (Table 3). The PSFs at different locations on the image in these bands have been carefully examined and characterized by fitting 2D Gaussians (see Galliano 2004) and are, to a high accuracy, symmetric. 


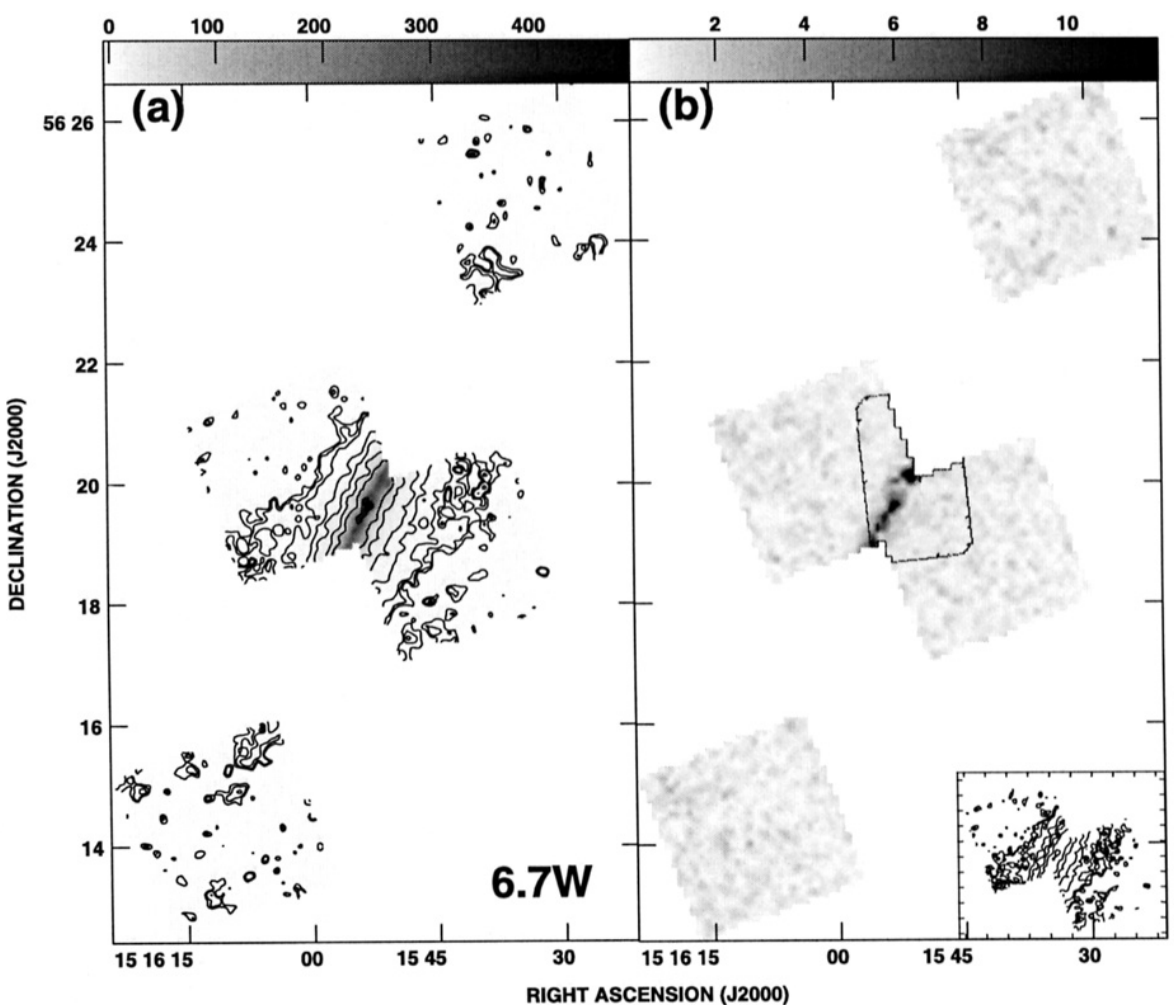

Fig. 6. As in Fig. 2, but for $6.7 \mathrm{~W}$ with contours at $0.0008\left(2 \sigma_{\text {map }}\right), 0.0012,0.002$, $0.005,0.010,0.030,0.10$, and $0.35 \mathrm{mJy}$ $\operatorname{arcsec}^{-2}$. The irregular region in b) denotes the area common to all maps.
Thus, the wings of the PSF should be completely negligible $7.2^{\prime \prime}$ beyond the projection of the disk. To check this, we took the $7.7 \mathrm{~N}$ image (Fig. 2), measured its semi-major axis (245"), and created an inclined $\left(86.5^{\circ}\right)$ thin disk model (see Irwin et al. 1999 for similar modelling). The resulting projected half-width perpendicular to the plane at the center of the galaxy is $15^{\prime \prime}$ and less elsewhere. This model was then used as a template to blank all emission outside of the inclined thin disk region from the Fig. 2 image. The resulting blanked image then contained only emission from the disk region. The blanked image was then convolved with a 7.2" Gaussian PSF to create an image consisting of disk plus extended emission due to the PSF only. This resulting map was then compared with the original image by taking the ratio of the two and determine the pencentage change per pixel that results. We find that, outside of the projection of the thin disk, all ratios are $<1 \%$ and the mean is $<0.05 \%$, verifying that the PSF contributes negligibly to the halo emission.

Secondly, we compared results between using the library flat field (as described in Sect. 2.2) and using a sky flat, formed from a portion of the image in which the sky is visible. The mean relative error between the two results over comparable regions is $4.5 \%$ and high latitude structure, most of which is in the region in which the sky flat could be formed, remains present. Moreover, in both images, artifacts, such as those at the edge of the field in Fig. 3a, cannot be seen.

Finally, we checked further into issues related to memory. Although the resulting map already has memory corrections in place including blanking the first frames of any scan, we applied a more aggressive scheme. This involved substantial blanking of the first time period when a new scan began, determining the median of the last 5 frames of the scan, pixel by pixel, and then fitting a slope to every pixel independently to align the flux of the other frames with this median. Some differences in structure were noted at a low level, however the high latitude emission remained.

\section{The MIR spectrum and contributors to the observed bands}

To interpret the emission in the observing bands, it is first necessary to understand what the various contributors are to the MIR emission. For this purpose, we provide ISOCAM CVF (circularly variable filter) spectra of regions in two other galaxies for comparison (see Fig. 7). The main spectrum is of the nucleus of the nearby galaxy, M 83, and the inset is of a quiescent region away from the starburst in M 82. Various features are labelled as well as the bands used in our observations.

There are two main contributors to the MIR spectrum: 1) strong spectral peaks due to very small grains or large molecules (PAHs) which, due to their small sizes (typically $\sim 0.001 \mu \mathrm{m}$ ) and thus, low heat capacity, are stochastically heated, undergoing wide temperature fluctuations covering $100 \mathrm{~s}$ of $K$ from absorption of a single photon (Leger \& Puget 1984; Sellgren et al. 1990; Allamandola et al. 1989). The primary emission bands, relevant to the wavelength range observed for NGC 5907, are located at 6.2, 7.7, 8.6, 11.3 and $12.7 \mu \mathrm{m}$ and can be seen as strong emission peaks in the spectrum. 2) a continuum composed of very small (of order $\sim 0.01 \mu \mathrm{m}$ ) grains (VSGs) as modeled in our galaxy by Désert et al. (1990). Depending on their size and the local radiation field, these grains could be stochastically heated or in thermal equilibrium. A modeled continuum is shown in the figure 


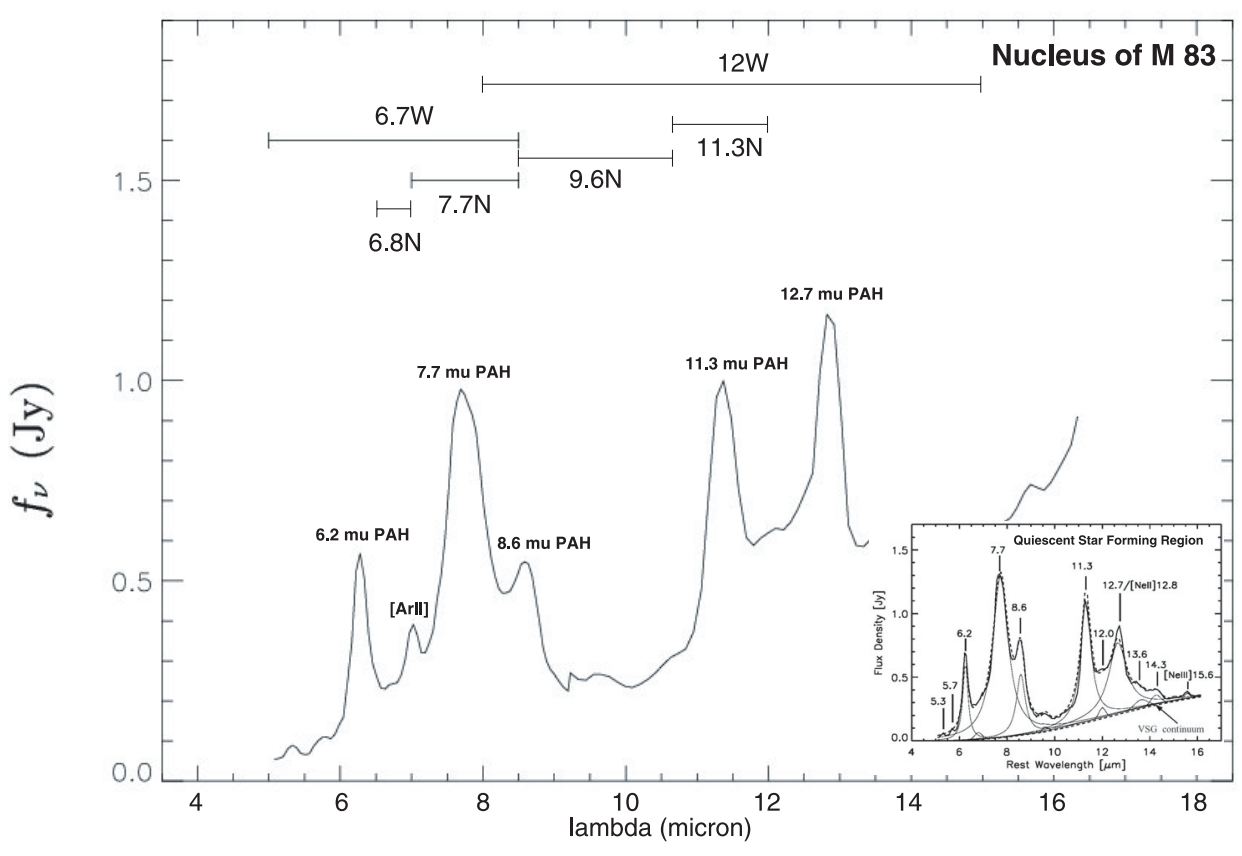

Fig. 7. Comparison MIR spectrum of the central position of $\mathrm{M} 83$ from the ISOCAM CVF observations of Vogler et al. (2005). The major features are labelled and the bands used in the observations of NGC 5907 are indicated at the top. Inset: MIR spectrum of a quiescent region in M 82, away from the central starburst, showing modeled components, from Laurent et al. (2000). inset, increasing in strength to longer wavelength. Finally, not shown in the figure, is a possible weak stellar contribution that, if present, would contribute at the low wavelength end of the spectrum, decreasing at higher wavelengths. Since we are able to make numerical estimates of this contribution from previous observations of NGC 5907 itself, we deal with this separately in Sect. 3.1.

Without doubt, the most overwhelming emission in the MIR band is due to PAH features. These features are ubiquitous in normal spirals and account for almost all of the MIR energy (e.g. Vogler 2005; Genzel \& Cesarsky 2000, and references therein). It is also now established that most normal spiral galaxies show qualitatively little difference in their spectra in the MIR and that the spectral shape is largely independent of star formation (Lu et al 2003). From higher resolution Galactic observations, we know that the VSGs (producing the dust continuum) peak in the nebular regions, while the PAHs peak outside the HII regions, in the photodissociation regions (PDRs) around molecular cores (e.g. Cesarsky et al 1996; Verstraete et al 1996). While the PAHs emit profusely in the disks of galaxies, excited predominantly by UV photons, they are also observed in the more diffuse ISM (e.g. Chan et al. 2001; Mattila et al. 1996) where optical photons are thought to be the source of excitation. Optical photons are also an explanation for the presence of PAHs in elliptical galaxies (Athey et al 2002; Xilouris et al 2004), where evolved stars are the primary stellar population. Moreover, in a study of 5 galaxies over a range of star forming activity, Haas et al. (2002) find a spatial correlation between PAHs and the cold dust distribution which is more widespread than around SFRs alone.

We thus expect the relative importance of the continuum to diminish in galaxies of lower star formation rate or in quiescent regions of galaxies. This is indeed observed in M 83 (the increasing continuum longward of $\lambda 14 \mu \mathrm{m}$ at the nucleus shown in Fig. 7 becomes much less prominent in the interarm regions) as well as other known quiescent star forming regions
(Fig. 7 inset). Thorough modeling of ISOCAM CVF spectra using Lorentizian profiles further supports the dominance of the PAH bands, greatly reducing the need for a strong continuum. (Boulanger et al. 1998). Indeed the three PAH features at $\lambda 6.2,7.7$, and $8.6 \mu \mathrm{m}$ in M 82 can be fit without any significant underlying continuum at all; a continuum contribution is fit with a simple linear curve, increasing with wavelength (Laurent et al. 2000). Vogler et al. (2005) also find that a continuum contributes only $\sim 5 \%$ for these three PAH bands. As this is less than a typical error bar on the observations, we consider the continuum contribution below these PAH features to be negligible.

Similar arguments apply to the ionic emission lines. In nuclear regions, a [NeII] emission line at $12.8 \mu \mathrm{m}$ has been observed and is blended with the $12.7 \mu \mathrm{m}$ PAH feature. However, in a sample of 69 nearby normal spiral galaxies with ISOCAM spectra, Roussel et al. (2001c) found that the contribution from the [NeII] line is negligible. The $\lambda 7.0 \mu \mathrm{m}$ [ArII] line, visible at the nucleus of M 83 (Fig. 7) is no longer seen in the interarm regions of this galaxy (Vogler et al. 2005).

The final possible MIR feature to be remarked upon is a broad silicate absorption feature at $9 \mu \mathrm{m}$ which has been detected in narrow central regions of very dusty starbursts and AGNs (e.g. Laurent et al. 2000; Tran et al. 2001; Sturm et al. 2000). This feature is not likely to be important toward the central regions of normal starbursts or in quiescent disks (e.g. Roussel et al 2001a, 2001b; Vogler et al 2005, Dale et al. 2001, also arguments in Spoon et al. 2004), even in the case of edgeon spiral galaxies (Mattila et al. 1999, for NGC891).

Therefore, the MIR spectrum of a quiescent galaxy like NGC 5907 can be expected to be dominated by the PAH emission bands, with a weak underlying continuum which becomes stronger at longer wavelengths. We thus interpret the emission in the various observed bands as follows:

6.8N: Lorentzian wings of the $\lambda 6.2 \mu \mathrm{m}$ and $\lambda 7.7 \mu \mathrm{m}$ PAH bands. 
7.7N: $\lambda 7.7 \mu \mathrm{m}$ PAH band.

9.6N: Lorentzian wings of the $\lambda 7.7 \mu \mathrm{m}, \lambda 8.6 \mu \mathrm{m}$, and $\lambda 11.3 \mu \mathrm{m}$ PAH bands plus possible minor contribution from a continuum. 11.3N: $\lambda 11.3 \mu \mathrm{m}$ PAH band plus possible continuum. 6.7W: Combined $\lambda 6.2, \lambda 7.7$, and part of the $\lambda 8.6 \mu \mathrm{m}$ PAH band emission.

12W: Broad band emission, equivalent to the IRAS $\lambda 12 \mu \mathrm{m}$ band, dominated by PAH features with likely continuum.

\subsection{The stellar contribution}

The stellar contribution to the MIR band is illustrated beautifully in Lu et al. (2003) for both a reddened and de-reddened case (their Fig. 6). These authors have obtained complete ISO spectra in the 2.4 to $5.9 \mu \mathrm{m}$ and 5.8 to $11.6 \mu \mathrm{m}$ bands for 45 galaxies finding, as have other authors, that the characteristic shape of the spectrum in these bands is extremely consistent, galaxy to galaxy. The stellar contribution can be approximated by a modified black body with a $\lambda^{-2}$ emissivity law. This behaviour for the stellar contribution also accurately describes the elliptical galaxies in their sample. Thus, we expect the stellar contribution in both the disk and halo regions of NGC 5907 to have a similar $\lambda$ dependence.

We have obtained the $K_{\mathrm{s}}$ band $(\lambda 2.159 \mu \mathrm{m})$ image for NGC 5907, from the 2MASS Large Galaxy Atlas (LGA, Jarrett et al. 2003), which is the band most likely to show only stars with negligible dust obscuration. This image was interpolated onto the same grid as our MIR images and then smoothed to 7.2 " resolution. The "data numbers" $(d n)$, in the FITS file were converted to Jy using the transformation, $f_{v}=$ $6.248 \times 10^{-6} d n \mathrm{Jy}^{2}$. As a check on our processing steps, we measured the total flux density of the source in the regridded, smoothed image, finding $f_{v}=1.322 \mathrm{Jy}$ which corresponds to $m_{K_{\mathrm{s}}}=6.76$. This agrees with the magnitude given in the LGA for NGC 5907 of $m_{K_{\mathrm{s}}}=6.76 \pm 0.02$. The resulting image has an rms noise of $\sigma=1.6 \mu \mathrm{Jy} \operatorname{arcsec}^{-2}$. From the emissivity law (following Lu et al.), we expect the stellar contribution, extrapolated into the MIR bands in which PAHs dominate to be $7.9 \%$ in the $7.7 \mathrm{~N}$ band, $3.7 \%$ at $11.3 \mathrm{~N}$, and $10 \%$ at $6.7 \mathrm{~W}$ of whatever value is in the $K_{\mathrm{s}}$ band at the relevant location. With this extrapolation, we created 3 maps from the $K_{\mathrm{s}}$ band image for the 3 bands dominated by PAH emission and subtracted each from the total emission map. These results are shown in the insets to Figs. 2, 4, and 6 with the same contour levels as the main maps. We then measured the global flux of the stellar-subtracted maps and compared this to the global flux of the main maps finding a difference of $5 \%, 2 \%$, and $2 \%$ for the $7.7 \mu \mathrm{m}, 11 \mu \mathrm{m}$, and $6.7 \mu \mathrm{m}$ maps, respectively (ignoring emission from the two outer frames in the latter case). Since the flux corrections are well within other error bars (Sect. 2) and the appearance of the maps is also close to the original non-subtracted maps, we consider the stellar contribution to be negligible and

\footnotetext{
2 This transformation uses the calibration, $m_{K_{\mathrm{s}}}=Z P-2.5 \log (d n)$ and $f_{v}=f_{v}(0) 10^{-0.4 m_{K_{\mathrm{S}}}}$ where $Z P=20.0704994$ is the zero point provided in the FITS header, and $f_{v}(0)=666.7 \mathrm{Jy}$, the latter from http://ssc. spitzer. caltech. edu/documents/cookbook/ html/cookbook.html
}

continue our discussion and analysis with respect to the unsubtracted maps.

\section{Results}

All maps along with the best estimated error maps are shown in Figs. 1 to 6 as described in Sect. 2.2. The error maps delineate the final field of view. Emission has been detected in every observing band as shown in these figures.

\subsection{Disk emission}

Emission is clearly detected along the disk of NGC 5907. In no individual band do we have full coverage of the entire galaxy. However, for the narrow bands, $6.8 \mathrm{~N}, 7.7 \mathrm{~N}, 9.6 \mathrm{~N}$, and $11.3 \mathrm{~N}$, we have full coverage of the south-east major axis and for $6.7 \mathrm{~W}$, small segments at both ends of the galaxy have been observed. In all cases, the emission extends along the optical disk with detections to the "end" of the optical disk in some cases.

The extent of the PAH emission, specifically, is illustrated by Fig. $8 \mathrm{a}$ in which we show the smoothed $7.7 \mathrm{~N}$ emission ( $\lambda 7.7 \mu \mathrm{m}$ PAHs) over the DSS optical image. It is clear from this overlay that the PAH emission extends all along the optical disk of the galaxy. It is further illustrated in Fig. 9 in which we show the smoothed, high sensitivity $6.7 \mathrm{~W}$ emission $(\lambda 6.2$, 7.7, and $8.6 \mu \mathrm{m}$ PAHs) superimposed on the HI total intensity map from Shang et al. (1998). PAH emission is seen very far out along the galaxy disk where the HI emission is also strong. The total extent of the PAH emission along the major axis is $11.0 \operatorname{arcmin}(\sim 35 \mathrm{kpc})$, as measured to the outermost detectable contiguous features on the unsmoothed map (cf. $41 \mathrm{kpc}$ for the optical disk, Table 1). However, there is also some evidence for real features farther out. For example, several emission peaks can be seen at the edges of the $6.7 \mathrm{~W}$ field of view on the south-east end of the major axis at RA $=15^{\mathrm{h}} 16^{\mathrm{m}} 12^{\mathrm{s}}$, Dec $=56^{\circ} 13^{\prime} 09^{\prime \prime}$. A channel-by-channel comparison (in velocity) of the HI distribution with the PAH emission, however, fails to show any clear correlation.

The lack of global correlation with $\mathrm{HI}$ is further illustrated in Fig. 10a in which we show major axis slices of the HI total intensity distribution, the $\mathrm{CO}$, and the smoothed $7.7 \mathrm{~N}$ emission. It is clear from this plot that the PAH emission follows the molecular gas distribution and not the atomic gas distribution. This was also found by Dumke et al. (1997) for the $\lambda 1.2 \mathrm{~mm}$ dust distribution as well as by Alton et al. (2004) for the $\lambda 850 \mu \mathrm{m}$ emission. Figure 10a shows two sets of peaks in the $\lambda 7.7 \mu \mathrm{m}$ distribution on either side of the nucleus, one at $\sim \pm 70^{\prime \prime}$ and the second at $\sim \pm 100^{\prime \prime}$. The CO comparison also show two peaks, interpreted by Dumke et al. (1997) to represent rings or spiral arms, but at somewhat offset radii of $\pm 60^{\prime \prime}$ and $\pm 120^{\prime \prime}$.

In Fig. 10b, we show a comparison between major axis cuts for our ISO $\lambda 7.7 \mu \mathrm{m}$ data and the $\lambda 850 \mu \mathrm{m}$ SCUBA data, both averaged over a minor axis extent of $16^{\prime \prime}$ and at the same spatial resolution. The shape of the PAH emission curve and the $\lambda 850 \mu \mathrm{m}$ emission curve, the latter which traces cool dust, are remarkably similar showing the same number and approximate, but not exact, positions of peaks on either side of the 

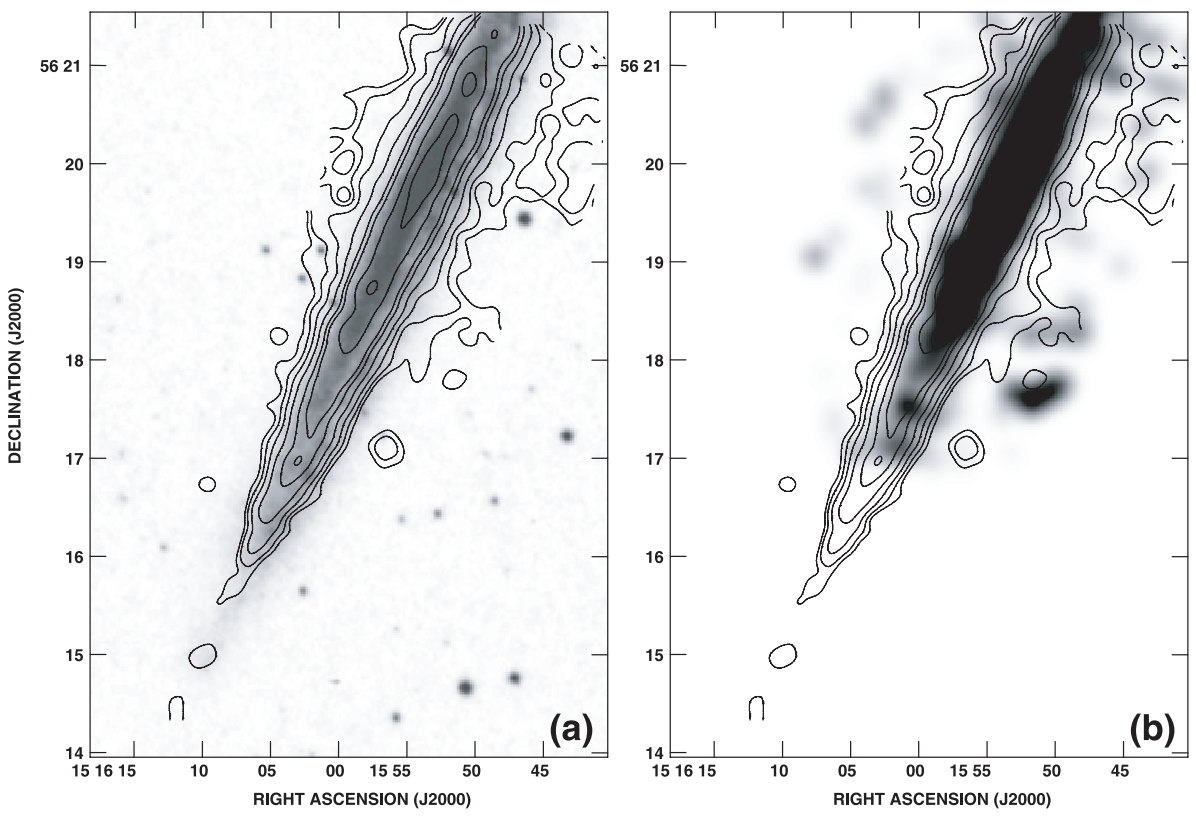

Fig. 8. a) The $7.7 \mathrm{~N}$ band image $(\lambda 7.7 \mu \mathrm{m}$ PAH band), smoothed to $12^{\prime \prime}$ resolution superimposed on the optical DSS image. Contours are at $4.6\left(2 \sigma_{\text {map }}\right), 7.5,13,25$, $50,75,200$, and $380 \times 10^{-3} \mathrm{mJy} \operatorname{arcsec}^{-2}$. b) The $7.7 \mathrm{~N}$ band image as in a) superimposed on a greyscale $\lambda 850$ SCUBA $\mu \mathrm{m}$ map from Alton et al. (2004) with a resolution of $16^{\prime \prime}$. Note that the SCUBA field of view ends at a Declination of $56^{\circ} 17^{\prime}$.

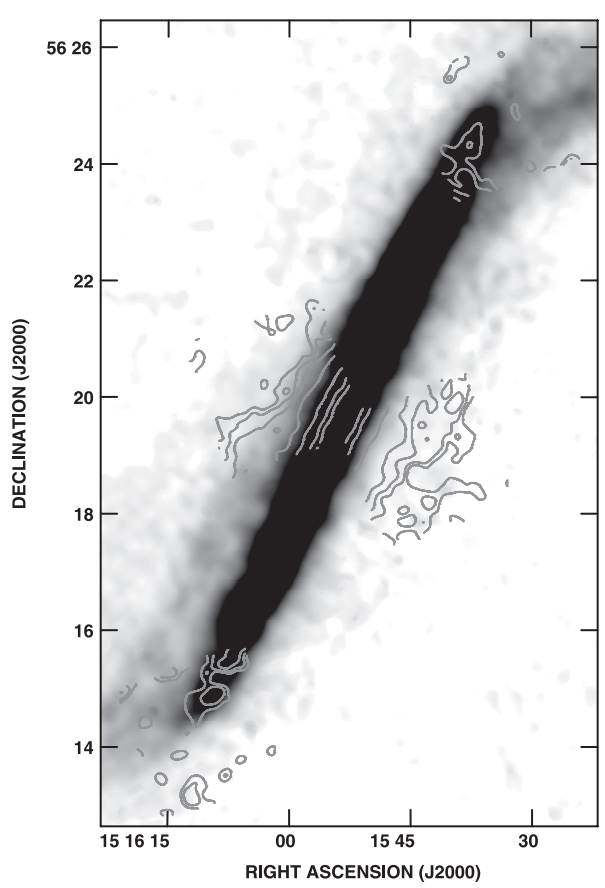

Fig. 9. The $6.7 \mathrm{~W}$ band image, smoothed to $12^{\prime \prime}$ resolution superimposed on the HI total intensity image (data from Shang et al. 1998). Contours are at $0.0004\left(2 \sigma_{\text {map }}\right), 0.0008,0.0015,0.003,0.01,0.04$, and $0.10 \mathrm{mJy} \operatorname{arcsec}^{-2}$.

nucleus. Positional offsets between the two different slices for 5 peaks range from zero to $19^{\prime \prime}$ with a typical value of $\sim 13^{\prime \prime}$. Given that the resolution is $16^{\prime \prime}$ and relative positional uncertainties between maps is of order 7 " (see caption), the positions of the peaks are likely consistent, though we cannot rule out the possibility of some displacement which might be detected with higher resolution observations. The line ratios from these curves (calculated though not plotted) are also consistent with the values of Haas et al. (2002) who found $\lambda 7.7 \mu \mathrm{m} / \lambda 850 \mu \mathrm{m}=$ 2 (1.8 to 2.2 for 5 galaxies). Their denominator is from SCUBA observations but the numerator represents the peak $\lambda 7.7 \mu \mathrm{m}$ flux rather than the average in the $7.7 \mathrm{~N}$ band as we have plotted. All of the emission in our $7.7 \mathrm{~N}$ band is from the $\lambda 7.7 \mu \mathrm{m}$ PAH line but the peak, if we could measure it directly, would be higher than the average. From Fig. 3, the increase should be of order a factor of $\approx 1.5$ which would put our ratio in even closer agreement with Haas et al. Therefore, overall, our results are consistent with a possible relationship between PAHs and the $850 \mu \mathrm{m}$ cool dust distribution.

Finally, there is excess $\lambda 7.7 \mu \mathrm{m}$ emission between radii of $-150^{\prime \prime}$ and $-250^{\prime \prime}$ (Fig. 10a) in comparison to the CO distribution. (Note that we cannot discern this in Fig. 10b because of the truncated field of view of the SCUBA map.) We believe that this excess is significant. Indeed, Dumke et al. (1997) also found an excess in the $\lambda 1.2 \mathrm{~mm}$ emission in comparison to $\mathrm{CO}$ over the same radii but on both the north and south sides of the nucleus. This shows that there is PAH emission as well as cool dust where there is little molecular gas. We will return to this point in Sect. 5 .

In Fig. 11 we show the broad band $12 \mathrm{~W}$ emission (equivalent to the IRAS $12 \mu \mathrm{m}$ band) superimposed on the DSS optical image. This MIR band should contain the highest contribution of continuum emission from VSGs compared to the other bands (Fig. 7, inset). The $12 \mathrm{~W}$ band has the highest spatial resolution of all observations $\left(1^{\prime \prime}\right)$ and reveals that the strongest ridge of MIR emission lies along the optical dust lane (note that the field of view does not cover the entire disk), indicating a good correlation between VSGs and the optically obscuring dust.

\subsection{High latitude emission}

A particularly striking result is the evidence for high latitude emission, especially in the $7.7 \mathrm{~N}$ band (Fig. 2 ), the $11.3 \mathrm{~N}$ band (Fig. 4), and the high-sensitivity beam-switched $6.7 \mathrm{~W}$ band (Fig. 6) which all trace PAH emission. The $11.3 \mathrm{~N}$ band in principle, can contain PAHs plus continuum, but the continnuum 

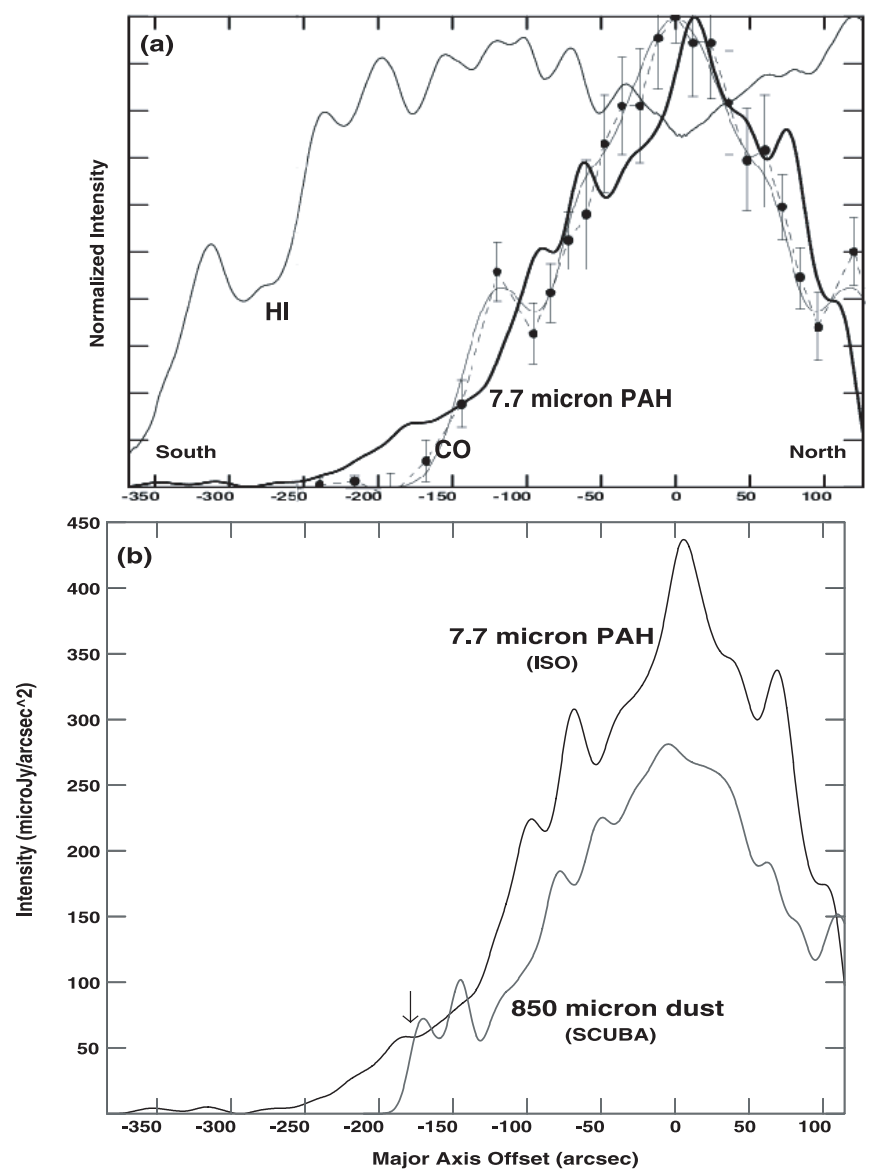

Fig. 10. Total intensity cuts along the major axis of NGC 5907. Positional uncertainties are of order $\pm 7^{\prime \prime}$. a) The HI total intensity (faint extended curve) from data of Shang et al. (1998), the CO distribution (faint solid plus dashed curves showing data points and error bars) from Dumke et al. (1997), and the $\lambda 7.7 \mu \mathrm{m}$ from this work (darkest curve), the latter smoothed to the same resolution as the HI data $\left(18.6^{\prime \prime} \times 17.8^{\prime \prime}\right.$ at $\left.\mathrm{PA}=-38^{\circ}\right)$. The $\mathrm{CO}$ resolution is $21^{\prime \prime}$ and the center point has been placed approximately at the $\mathrm{CO}$ center of RA $(\mathrm{J} 2000)=$ $15^{\mathrm{h}} 15^{\mathrm{m}} 53.8^{\mathrm{s}}, \operatorname{Dec}(\mathrm{J} 2000)=56^{\circ} 19^{\prime} 37^{\prime \prime}$. b) The $\lambda 7.7 \mu \mathrm{m}$ PAH emission from the $7.7 \mathrm{~N}$ band, smoothed to $16^{\prime \prime}$ resolution shown with the $\lambda 850 \mu \mathrm{m}$ SCUBA emission at the same resolution. Curves and intensities represent an average over a $16^{\prime \prime}$ swath parallel to the major axis. The arrow marks the edge of the SCUBA field of view.

should be negligible so far from star forming regions. Thus it is clear that PAHs exist far from the plane of NGC 5907, in the case of $6.7 \mathrm{~W}$, as far as $6.5 \mathrm{kpc}\left(2^{\prime}\right)$ from the plane, though $\sim 3 \mathrm{kpc}$ is more typical. Even the $6.8 \mathrm{~N}$ band (Fig. 1), which should consist only of emission from PAH band wings, shows some evidence for features away from the plane. Given the galaxy's edge-on orientation $\left(86.5^{\circ}\right.$, Table 1$)$, the correction for galaxy inclination is negligible. For example, for a PAH disk radius of $250^{\prime \prime}$ (Fig. 10), the semi-minor axis is only $15.3^{\prime \prime}$ in a thin disk model. Moreover, the $\mathrm{CO}$ semi-minor axis is only $\sim 4$ arcsec if we use the $H W H M$ of 200 pc modeled by Dumke et al. (1997). These features resemble the extra-planar emission seen in other galaxies, (see, e.g. the radio continuum features in NGC 5775, Lee et al. 2001) and some are similarly arc-like in appearance (e.g. the east side of the $\lambda 7.7 \mu \mathrm{m}$ emission in

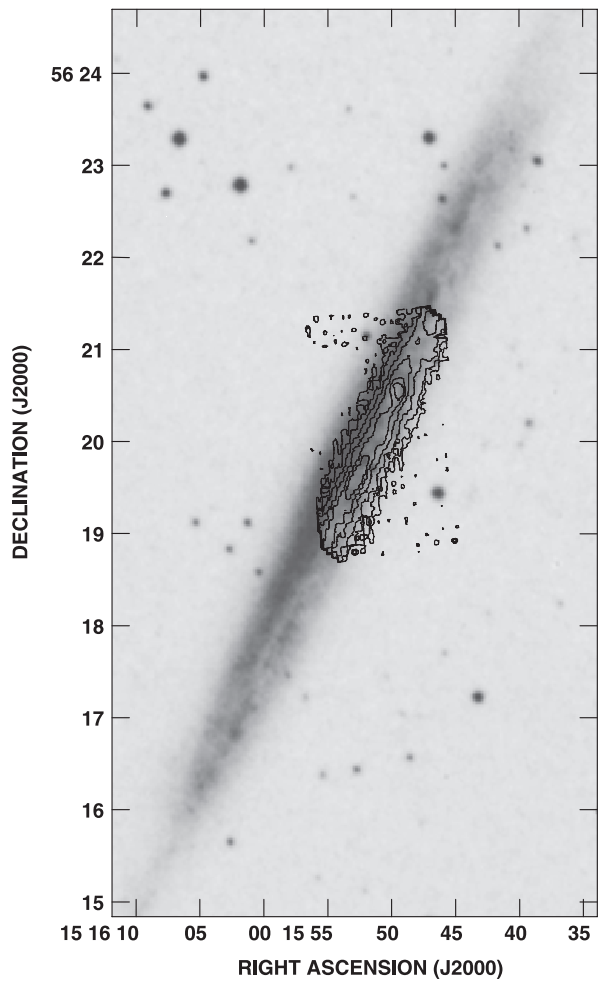

Fig. 11. The IRAS band-equivalent image, $12 \mathrm{~W}$, (contours) over the Digitized Sky Survey (DSS) optical image. Contours are at 0.028, $0.050,0.10,0.18,0.28$, and $0.36 \mathrm{mJy} \mathrm{arcsec}^{-2}$. The $12 \mathrm{~W}$ field of view does not extend over the whole optical image (see Fig. 5b). The known faint stellar ring (Sect. 1.1), which cannot be discerned in this image, intersects the disk at declinations of $56^{\circ} 23.4^{\prime}$ and $56^{\circ} 16.8^{\prime}$ in projection (Zheng et al. 1999).

Fig. 2). Recall also (Sect. 1.1) that the stellar scale height has been measured to be $0.49 \mathrm{kpc}\left(9^{\prime \prime}\right)$. Note that the narrow bands, $7.7 \mathrm{~N}$ and $11.3 \mathrm{~N}$, show more structure than the wide band, $6.7 \mathrm{~W}$ which may result from the ability of these bands to isolate a single PAH line. Although structural details may vary, both narrow bands show high latitude features at approximately the same locations (see also Sect. 2.3).

Given that the beam-switched $6.7 \mathrm{~W}$ band observations are our most sensitive and also the fact that this band is dominated by several PAH emission features, we have taken the original, unsmoothed $6.7 \mathrm{~W}$ data shown in the central regions of Fig. 6 and averaged the emission in strips parallel to the major axis. The resulting minor axis profile is shown in Fig. 12. The wings on this averaged profile approach the noise between $100^{\prime \prime}$ and $150^{\prime \prime}(5.3 \mathrm{kpc}$ to $8.0 \mathrm{kpc})$ and the line half-width at the $3 \sigma$ level is $\sim 95^{\prime \prime}(5 \mathrm{kpc}$ ). We fit various functional forms to the profile up to 3 components in total, including Gaussians, Lorentzians, Voigt, combinations of these forms and a combination of Gaussian + exponential. The best fit results were achieved by 3 Gaussians or 1 Gaussian plus 1 Voigt profile, which resulted in equivalent residual rms values. However, since the Voigt profile does not have easily identifiable parameters, we show the fits (Fig. 12) for the Gaussian combinations only and provide tabular information (Table 4) for these combinations as well as the Gaussian + exponential for comparative purposes. The broadest Gaussian scale length is 

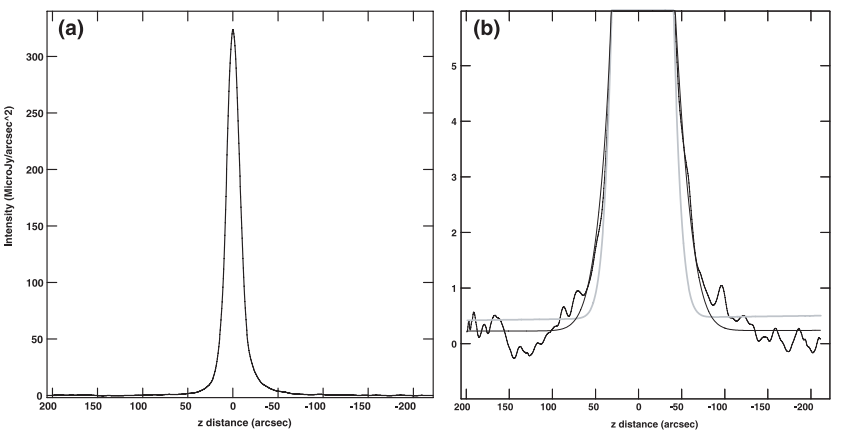

Fig. 12. Average profile of the PAH emission as a function of $z$ from the high sensitivity 6.7W data (Fig. 6). Negative values of $z$ represent the western side of the major axis. a) Plot of the data over the full range of intensity. b) Plot of the data and two of the models over only the lowest $1.7 \%$ of the total intensity. The dark solid curve shows the data with the noise, at an rms level of $2 \times 10^{-4} \mathrm{mJy} \mathrm{arcsec}^{-2}$, now obvious. The grey curve shows the best fit 2-component Gaussian model and the narrower dark solid curves shows the best fit 3-component Gaussian model. See Table 4 for the parameters of the Gaussian fits.

$65.3^{\prime \prime}(3.5 \mathrm{kpc})$. Increasing the number of components would reduce the residual rms further but such a situation is likely not physical. The profile, in general, consists of a central Gaussian component plus broad wings. Also, since the high latitude emission is highly structured (Fig. 6), the scale length of the broad component will vary with position. Nevertheless, it is clear that an extended halo or thick disk of PAH emission is present in NGC 5907 with a characteristic scale height of 3.5 to $5 \mathrm{kpc}$.

Given the apparent correlation between the ISO $\lambda 7.7 \mu \mathrm{m}$ emission and the SCUBA $\lambda 850 \mu \mathrm{m}$ emission in the disk (Sect. 4.1), it is of interest to see if this correlation extends to the halo region. Figure $8 \mathrm{~b}$ shows a comparison between these bands. Note that the $\lambda 850 \mu \mathrm{m}$ field of view is truncated at a Declination of $56^{\circ} 17^{\prime}$ and the $7.7 \mathrm{~N}$ map is truncated on the eastern side. Note also that the greyscale showing apparent emission away from the plane in the SCUBA data is all below the $3 \sigma$ level (Alton et al. 2004, their Fig. 1) with the exception of the "detached" feature at RA $=15 \mathrm{~h} 15 \mathrm{~m} 52 \mathrm{~s}$, Dec $=$ $56^{\circ} 17^{\prime} 45^{\prime \prime}$. Indeed, Alton et al. find an exponential scaleheight of only $0.11 \mathrm{kpc}\left(2^{\prime \prime}\right)$ for the $\lambda 850 \mu \mathrm{m}$ map. If the $\lambda 850 \mu \mathrm{m}$ emission existed in the halo at the same fraction of the indisk emission as the PAHs, then the $\lambda 850 \mu \mathrm{m}$ emission should have been detected in the halo above the noise of the Alton et al. map. This suggests that either the large grains are underrepresented in the halo in comparison to the PAHs, or are under-emitting.

The broad band $12 \mathrm{~W}$ emission shows very little vertically extended emission (Figs. 5, 11) in comparison to the PAH bands (cf. Figs. 2 and 4). This is likely due to the fact that the dynamic range in the $12 \mathrm{~W}$ band is only 10 to $17 \%$ as great as in the $7.7 \mathrm{~N}$ and $11.3 \mathrm{~N}$ bands (Table 3 ). For example, the extended vertical emission seen in Figs. 2 and 4, if present in Fig. 5 at the same relative brightness with respect to the maximum disk emission, would mostly fall at the level of the noise or lower. One obvious feature is visible at the edge of the field of view on the north, extending to the east. (The optical feature within this extension appears to be a foreground star.) The reality of the large scale feature is not clear but its "footprints" close to the disk have counterparts in the PAH bands (e.g. Fig. 4) suggesting that the PAH contribution within this band may be responsible for it.

\subsection{Flux densities and band ratios}

In no case do we have complete coverage of the galaxy so global flux densities cannot be obtained. However, we have determined the flux density for each of the fields displayed. These are listed in Table 5. Since the $6.8 \mathrm{~N}$ through $11.3 \mathrm{~N}$ bands have the same field of view, their fluxes can be compared directly. There is one common field for all maps, however, which is outlined in Fig. 6b. Thus, we also calculate fluxes for this common region (Table 5) and compare these results for all bands. The resulting flux ratios are provided in Table 6 with some comparative values, where known, also given.

The $6.7 \mathrm{~W}: 12 \mathrm{~W}$ ratio is interesting since it is significantly higher than the values given by Dale et al. (2001). This ratio represents a comparison between a broad ISOCAM band that contains mainly 3 PAH bands (Sect. 3 ) with the broader $12 \mu \mathrm{m}$ IRAS-equivalent band which may contain both PAHs and continuum. Dale et al. (2001) determined average values of this ratio, using the IRAS $12 \mu \mathrm{m}$ flux itself, for normal galaxies in different $f_{60} / f_{100}$ bins. The IRAS flux ratio, $f_{60} / f_{100}$ is a measure of the heating of classical grains in temperature equilibrium. As such, it is widely used as a measure of star formation activity, with an increasing ratio implying greater dust heating (Helou 1986; Dale et al. 2000, 2001). As the SFR increases $\left(f_{60} / f_{100}\right.$ increases), the $6.7 \mathrm{~W} /($ broad-band $12 \mu \mathrm{m}$ ) ratio decreases, reflecting the fact that a) the contribution of a hot dust continuum increases with SFR and b) PAHs are expected to be destroyed in regions of high heating intensity such as active star forming regions (Dale et al. 2001 and references therein). The $f_{60} / f_{100}$ ratio for NGC $5907(0.24$, Table 1) places it at a SFR that falls below all of the galaxies of the Dale et al. sample. Moreover, the $f_{60} / f_{100}$ ratio for NGC 5907 could be even lower than that quoted in Table 1, i.e. 0.21 from earlier ISO data, Bendo et al. (2002). Thus, the high 6.7W:12W ratio for NGC 5907 (Table 6) is likely consistent with the data from other galaxies in that it would represent an extension to lower SFR.

The ratios for the bands $6.8 \mathrm{~N}$ through $11.3 \mathrm{~N}$ (Table 6) all have the same field of view and so can be compared directly. (Note also, that all results for the "field" and for the "common" regions agree within errors.) For these bands, we make a comparison to the galaxy, M 83, for which similar observations have been made. NGC 5907 is not perfectly identical to M 83 in its global properties (Table 1). The two galaxies are of similar morphological types and have similar global star formation rates, infrared luminosities, and modelled temperatures. However, M 83 is physically smaller than NGC 5907. Thus, NGC 5907 contains an order of magnitude more dust but M 83 is the more active galaxy when SFR per unit area is determined. Nevertheless, the shapes of the different spectra in M 83 (see Vogler et al. 2005 and Fig. 7) are remarkably constant as a function of location in that galaxy, i.e. as a function of differing local SFR, the main differences being increased 
Table 4. $6.7 \mathrm{~W}$ z profile.

\begin{tabular}{lcccc}
\hline \hline Data or type of fit & Component No. & $\begin{array}{c}\text { Peak intensity } \\
\left(\mathrm{mJy} \mathrm{arcsec}^{-2}\right)\end{array}$ & $\begin{array}{c}F W H M^{a} \\
(\operatorname{arcsec})\end{array}$ & $\begin{array}{c}\text { Residual rms } \\
\left(\mathrm{mJy} \mathrm{arcsec}^{-2}\right)\end{array}$ \\
\hline Data & 1 & & & $0.20 \times 10^{-3}$ \\
1-component Gaussian & 1 & 0.315 & 18.3 & $3.96 \times 10^{-3}$ \\
2-component Gaussian & 2 & 0.287 & 16.2 & \\
Gaussian + Exponential & 1 & 0.0376 & 43.2 & $1.07 \times 10^{-3}$ \\
& 2 & 0.264 & 16.6 & \\
3-component Gaussian & 1 & 0.065 & $16.5^{b}$ & $1.11 \times 10^{-3}$ \\
& 2 & 0.257 & 15.5 & \\
& 3 & 0.0564 & 27.2 & \\
& 3 & 0.00131 & 65.3 & $0.86 \times 10^{-3}$ \\
\hline
\end{tabular}

${ }^{a}$ Unless otherwise indicated.

${ }^{b}$ Exponential scale length.

Table 5. Flux densities.

\begin{tabular}{|c|c|c|}
\hline$\overline{\text { Band }}$ & $\begin{array}{c}\text { Flux density (field) }{ }^{a} \\
(\mathrm{mJy})\end{array}$ & $\begin{array}{l}\text { Flux Density (Common })^{b} \\
(\mathrm{mJy})\end{array}$ \\
\hline $6.8 \mathrm{~N}$ & $1170 \pm 97$ & $519 \pm 42$ \\
\hline $7.7 \mathrm{~N}$ & $2170 \pm 141$ & $918 \pm 60$ \\
\hline $9.6 \mathrm{~N}$ & $919^{c} \pm 66$ & $360 \pm 26$ \\
\hline $11.3 \mathrm{~N}$ & $2071 \pm 116$ & $828 \pm 46$ \\
\hline $12 \mathrm{~W}$ & $940 \pm 38$ & $557 \pm 57$ \\
\hline $6.7 \mathrm{~W}$ & $666 \pm 22$ & $591 \pm 20$ \\
\hline
\end{tabular}

a Flux density determined over the entire field shown in Fig. 1a through Fig. 6a. The error (here and throughout) is calculated via $\sqrt{\Sigma \sigma_{i}^{2}}$ over the map pixels, $i$, where $\sigma_{i}$ is the quadratic sum at each pixel of $\sigma_{\text {rms }}$, the Transient rms, the $1 \sigma$ sky error and the calibration error (see Table 3).

${ }^{b}$ Flux density determined over the region that is common to all maps and outlined in Fig. 6b.

${ }^{c}$ Excluding the 3 small regions with residual errors (see Sect. 2.3).

contributions of continuum, especially longwards of $14 \mu \mathrm{m}$ (which we sample only marginally in NGC 5907 in the $12 \mathrm{~W}$ band). For M 83, the main differences between spectra can be seen as an increased continuum contribution from hot grains which become more prominent towards the nucleus. Thus, we provide the $6.8 \mathrm{~N}$ through $11.3 \mathrm{~N}$ ratios for $\mathrm{M} 83$ for the inner, hotter, $1^{\prime}$ radius region as well as the outer, cooler, region between radii of $1^{\prime}$ and $3^{\prime}$ in Table 6 . Given the estimated errors on the various ratios, there is agreement between all values for M 83 and those of NGC 5907, although the most variation is seen for $9.6 \mathrm{~N}$. The band, $9.6 \mathrm{~N}$, measures a mixture of PAH wings plus possible hot continuum. The $9.6 \mathrm{~N}: 7.7 \mathrm{~N}$ ratio for NGC 5907 is somewhat low in comparison to the nucleus of M 83 but agrees with the result for the interarm region of M 83 . This is again quite consistent with the low SFR for NGC 5907.

The Dale et al. comparison values for $6.7 \mathrm{~W}: 12 \mathrm{~W}$ are globally determined values. Our data allow us to further examine this ratio as a function of projected distance from the major axis, as shown in Fig. 13. In Fig. 13a, we show the increasing $6.7 \mathrm{~W}: 12 \mathrm{~W}$ ratio with projected distance from the major axis. Given that this ratio can only be determined over a restricted area of the "common" region due to signal-to-noise considerations, this may reflect either an increase in this ratio with $z$ height or an increase with distance from the center of the galaxy since the region shown does not extend beyond the projected optical disk. In either case, this is likely due to an increase in the distance from star forming regions and therefore a decrease in the contribution of the hot continuum that would be expected in the $12 \mathrm{~W}$ band. In Fig. 13b, we show the ratio of the emission in two PAH bands: $11.3 \mathrm{~N} / 7.7 \mathrm{~N}$. In this case, the ratio can be determined to much higher latitude. The maximum change in ratio is of order 2.4 with the in-disk value lower. Given that more continuum, if present, is expected in the $11.3 \mathrm{~N}$ band than the $7.7 \mathrm{~N}$ band in the disk, then the in-disk ratio could be lower than shown. As indicated above, the global ratio of these two bands is quite consistent with what is seen in other galaxies so we may be observing a real variation in the 11.3/7.7 $\mu \mathrm{m}$ PAH ratio between the changing environments of the disk and halo. The nature of this variation is unclear and the exact magnitude will require the acquisition of spectra and modelling. However, we note that the variation seen here is not inconsistent with the variations between PAH feature strengths observed for regions in our own Milky Way, i.e. of order a factor of 5 (see Peeters et al. 2002).

\subsection{Radio continuum correlation?}

Vogler et al. (2005) find a good spatial correlation between the $\lambda 6 \mathrm{~cm}$ radio continuum emission and the $6.7 \mathrm{~W}$ (largely PAH) MIR emission in M 83, and Brar et al. (2003) also find a good correlation between the $\lambda 850 \mu \mathrm{m}$ emission and the $617 \mathrm{MHz}$ radio continuum emission in the edge-on galaxy, NGC 5775. These results, which link the MIR or sub-mm with the radio continuum, are reminiscent of the well-known FIR-radio correlation whose origin is not fully understood but is thought to relate to star formation in galaxies (see Groves et al. 2003, and references therein). Thus, a comparison of our ISO results with radio data is of interest. The best available radio data sets are those of the NRAO VLA Sky Survey (NVSS) at $1420 \mathrm{MHz}$ (Condon et al. 1998) and the Westerbork Northern Sky Survey (WENSS) at $327 \mathrm{MHz}$ (Rengelink et al. 1997). The radio emission in the in the VLA Faint Images of the Radio 
Table 6. Flux density ratios.

\begin{tabular}{|c|c|c|c|}
\hline Bands $^{a}$ & Ratio or relation & Galaxy or region & Ref. \\
\hline 6.7W:12(IRAS) & {$\left[0.52_{-0.15}^{+0.22}\right]: 1^{b}$} & 8 galaxies & Dale et al. (2001) \\
\hline $6.7 \mathrm{~W}: 12 \mathrm{~W}$ & {$[1.06 \pm 0.16]^{c}: 1$} & Common $^{d}$ & This work \\
\hline $6.8 \mathrm{~N}: 7.7 \mathrm{~N}: 9.6 \mathrm{~N}: 11.3 \mathrm{~N}$ & {$[0.57 \pm 0.08]: 1:[0.39 \pm 0.05]:[0.90 \pm 0.11]$} & Common $^{d}$ & This work \\
\hline $6.8 \mathrm{~N}: 7.7 \mathrm{~N}: 9.6 \mathrm{~N}: 11.3 \mathrm{~N}$ & {$[0.54 \pm 0.08]: 1:[0.42 \pm 0.06]:[0.95 \pm 0.12]$} & Field $^{d}$ & This work \\
\hline $6.8 \mathrm{~N}: 7.7 \mathrm{~N}: 9.6 \mathrm{~N}: 11.3 \mathrm{~N}$ & $0.49: 1: 0.59: 1.12^{e}$ & $\operatorname{M~} 83\left(R \leq 1^{\prime}\right)^{f}$ & Vogler et al. (2005) \\
\hline $6.8 \mathrm{~N}: 7.7 \mathrm{~N}: 9.6 \mathrm{~N}: 11.3 \mathrm{~N}$ & 0.55:1:0.48:0.95 & $\operatorname{M} 83\left(1^{\prime} \leq R \leq 3^{\prime}\right)^{f}$ & Vogler et al. (2005) \\
\hline
\end{tabular}

${ }^{a}$ The center wavelengths of the bands is given below each sequence that is the same.

${ }^{b}$ Average of 8 galaxies in a flux ratio, $f_{60} / f_{100}$, bin from 0.28 to 0.35 (Dale et al. 2001).

${ }^{c}$ This error includes an additional 5\% error to account for variations in orbit (Sect. 2.3).

${ }^{d}$ Common or Field region as quoted in Table 5.

${ }^{e}$ Errors (estimates only) are believed to be of order $20 \%$.

${ }^{f} R$ is the projected radial distance from the center of the galaxy.
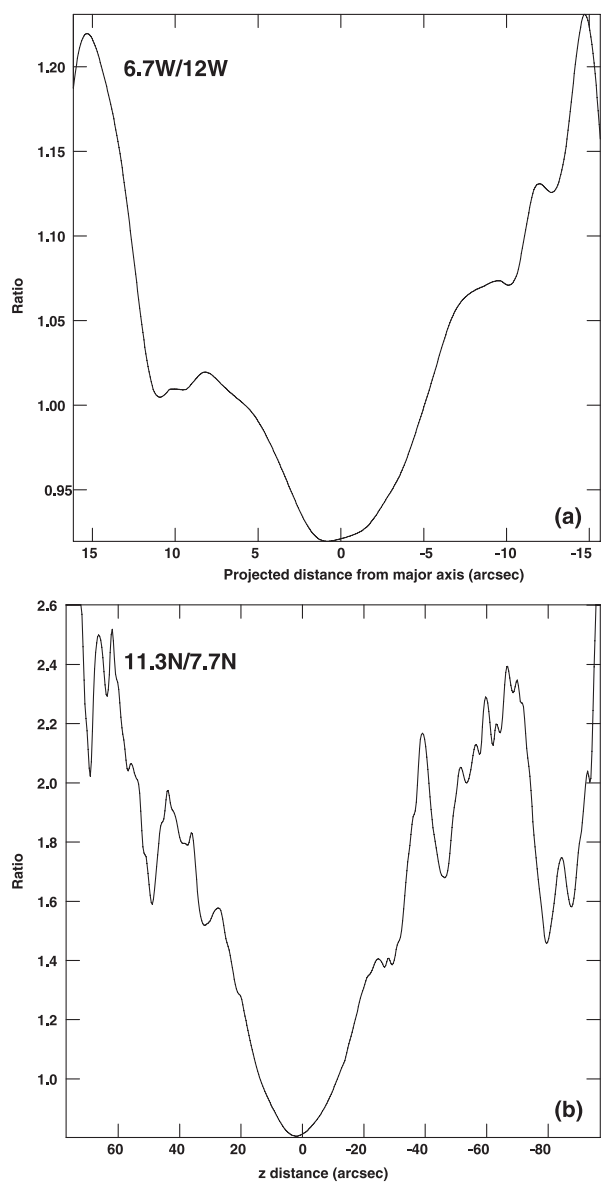

Fig. 13. a) Profile of the $6.7 \mathrm{~W} / 12 \mathrm{~W}$ ratio from the common region only (see Fig. 6b) as a function of projected distance from the major axis. The values are averages over $71^{\prime \prime}$ strips parallel to the major axis. b) The $z$ profile of the $11.3 \mathrm{~N} / 7.7 \mathrm{~N}$ ratio for the fields shown in Figs. 2 and 4 , but averaged over $320^{\prime \prime}$ strips. Note that the $z$ extent is much larger than in a).

Sky at Twenty-Centimeters survey (FIRST) is too faint for comparison (but see note below). In Fig. 14, we show the $7.7 \mathrm{~N}(\lambda 7.7 \mu \mathrm{m} \mathrm{PAH})$ emission, which is unlikely to contain any significant continuum component, in comparison to these two radio images. The strong radio source at the end of the south-eastern major axis (actually a double) is believed to be from a background source (Dumke et al. 2000). There are significant differences between the fields of view and resolutions of the ISO and radio data sets which make comparison difficult, and smoothing the $7.7 \mathrm{~N}$ emission results in confusion and truncations near the field edges. In addition, details in the structure of the $7.7 \mathrm{~N}$ map at low emission levels may be approximate. Thus any apparent correlations are tentative and are presented so that higher resolution data in the future might be compared to these results. In particular, a radio continuum extension on the west side of the major axis at $\mathrm{RA} \approx 15 \mathrm{~h} 15 \mathrm{~m} 45 \mathrm{~s}$, Dec $\approx$ $56^{\circ} 20^{\prime}$ shows extended PAH emission (though the ISO field of view also truncates here). South of this feature is another at $\mathrm{RA} \approx 15 \mathrm{~h} 15 \mathrm{~m} 50 \mathrm{~s}$ Dec $\approx 56^{\circ} 18^{\prime}$. On the east side of the major axis, there is a feature at $\mathrm{RA} \approx 15 \mathrm{~h} 16 \mathrm{~m} \mathrm{00s} \mathrm{Dec} \approx 56^{\circ} 20^{\prime}$. These features in the radio are more easily seen in the $327 \mathrm{MHz}$ image and at least the northernmost extension on the west side can also be seen in a $20 \mathrm{~cm}$ image of Dumke et al. (2000). We are not able to determine correlations along the disk due to the limited ISO field of view and resolution and also because of confusion with the strong background source.

Finally, we note that there is a previously unreported point source in the FIRST data with coordinates, RA $(J 2000)=$ $15 \mathrm{~h} 15 \mathrm{~m} 49.10 \mathrm{~s}, \operatorname{Dec}(\mathrm{J} 2000)=56^{\circ} 20^{\prime} 27.1^{\prime \prime}$, which is displaced $38^{\prime \prime}$ to the west and $43^{\prime \prime}$ to the north of the nucleus ( 2.0 and $2.3 \mathrm{kpc}$, respectively, in projection) with a flux density of $3.1 \mathrm{mJy}$. This flux density corresponds to a spectral power of $4.5 \times 10^{19}$ Watts/ $\mathrm{Hz}$ which is intermediate between that of Cas A and the brightest supernova remnant in M 82 (see Irwin et al. 2000) and is therefore likely a supernova remnant or remnants.

\section{Discussion}

The mid-IR emission in NGC 5907 is consistent with NGC 5907 as a low SFR galaxy. As described in Sect. 4.3, this galaxy is "PAH-rich" and "hot-dust-poor". Our flux ratios are consistent with those measured in other galaxies at the low end of the range of SFR and are also consistent with those of Vogler et al. (2005) for M 83 for the regions between spiral arms. For M 83 , the continuum contributes no more than $\approx 10 \%$ of the total MIR 4 to $18 \mu \mathrm{m}$ emission with $\approx 90 \%$ due to PAHs in these 


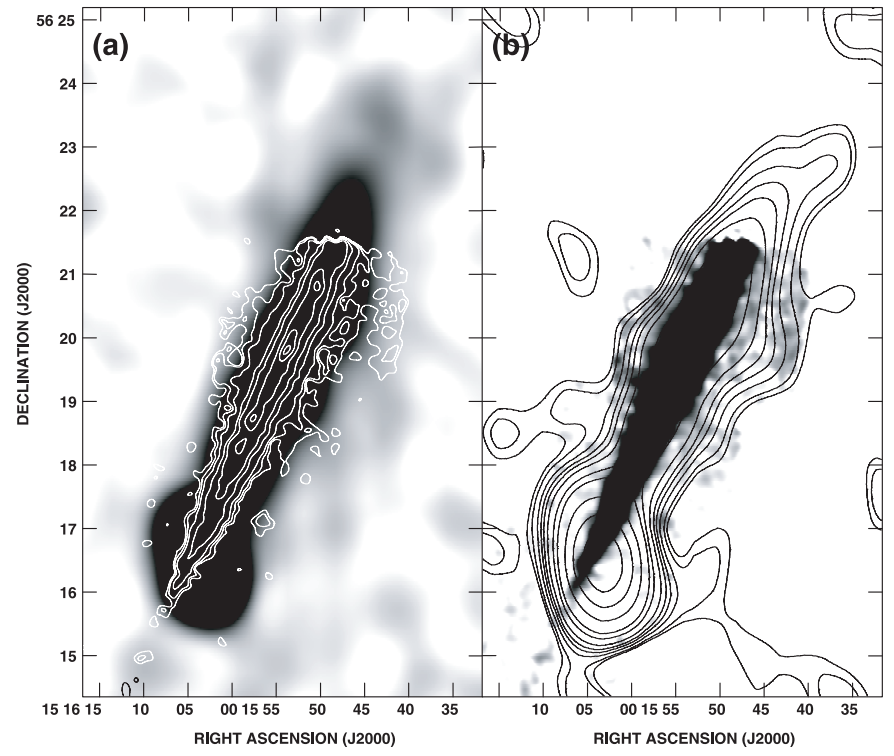

Fig. 14. a) The $7.7 \mathrm{~N}$ band image, with contours and field of view as shown in Fig. 2, superimposed on the NVSS $\lambda 20 \mathrm{~cm}$ radio continuum image with low level emission enhanced. The greyscale ranges from $0.01(\approx 1 \sigma)$ to $4 \mathrm{mJy}$ beam $^{-1}$, with a beam $45^{\prime \prime}$ in size. The strong source at the south-east end of the major axis is believed to be a background source (Dumke et al. 2000). b) WENSS $327 \mathrm{MHz}$ image (contours) superimposed on a greyscale $7.7 \mathrm{~N}$ band image. Contours are at $4.5(1.5 \sigma), 6,9,12,15,20,30,40,70,110$, and $160 \mathrm{mJy} \mathrm{beam}^{-1}$ with a beam size of 54" in Right Ascension and 64.9" in Declination. The greyscale ranges from 0.003 to $0.02 \mathrm{mJy} \operatorname{arcsec}^{-2}$.

regions. Although we do not have CVF data for NGC 5907, all other indicators suggest that the situation is likely comparable.

It is quite clear that the PAH distribution follows that of the CO (Fig. 10). Both the $\lambda 850 \mu \mathrm{m}$ and $\lambda 1.2 \mathrm{~mm}$ cool dust also follow the $\mathrm{CO}$ distribution, and the $\mathrm{PAH}$ and $\lambda 850 \mu \mathrm{m}$ are spatially well correlated within the disk of NGC 5907. This suggests that both the PAHs and larger grains are associated with the molecular gas component. PAHs are well known to trace classical photodissociation regions (PDRs) of molecular clouds which are excited by far-UV photons from a hot stellar population in our own Galaxy (see Sect. 3). However there is also evidence that the PAHs are extended radially in comparison to $\mathrm{CO}$ both from our results as well as the cool $1.2 \mathrm{~mm}$ dust distribution of Dumke et al. (1997). The cool dust and PAH emission both extend to radii of $\sim 250^{\prime \prime}$ in comparison to $\sim 175^{\prime \prime}$ for CO. While there may be some weak CO present, the PAH and cool dust distributions show an excess with respect to the $\mathrm{CO}$ at large radii in comparison to smaller radii. These results suggest that, while PAHs are strongly associated with the molecular gas, a more widespread distribution, likely associated with cool HI, is also present but at a much weaker level of emission.

Haas et al. (2002) also find a correlation between PAHs and cold dust, the latter as measured by the SCUBA $\lambda 850 \mu \mathrm{m}$ continuum emission, in galaxies. These authors suggest that PAH carriers are widespread and correlate with large dust grains and neutral molecular clouds, but require a minimal radiation field in order to be excited, thus explaining global correlations between SFR and PAH emission as well as the fact that PAHs are distributed more widely than the SF regions alone. This is also consistent with Peeters et al. (2004) who suggest that PAHs may be better tracers of B stars, which dominate the Galactic stellar energy budget, rather than as a tracer of massive star formation. As indicated in Sect. 3, the PAHs in our Galaxy peak outside of HII regions but are also profuse emitters in the diffuse ISM of galaxy disks, where the excitation may be from optical photons from an older population. These studies therefore support the association of PAHs with more extended neutral atomic gas. Tacconi-Garman et al. (2005) find that the global distribution of PAHs in NGC 253 and NGC 1808 follows the starburst activity, but on small scales, there is little correspondence (correlation or anti-correlation) with star forming regions. It may well be that PAHs are widespread and excited by both UV photons from a hot stellar population as well as optical photons from the more widespread stellar population from which the interstellar radiation field (ISRF) originates. The fact that they correlate globally with the $\mathrm{CO}$ distribution would then be a statement that there are more UV photons available at the appropriate wavelength and also that there is a greater concentration of dust and PAHs in regions of higher density molecular clouds. In regions (or galaxies) with very high SFRs the PAHs will be destroyed in comparison to the VSGs; this is supported by observations of M 83 (Vogler et al. 2005) and of starbursts and AGNS (e.g. Sturm et al. 2000).

An important result is the presence of PAH emission to very high latitude, with scale heights of order 3.5 to $5 \mathrm{kpc}$ and up to $6.5 \mathrm{kpc}$ in some locations. By comparison, the CO FWHM in the $z$ direction is only $\sim 8^{\prime \prime}=426 \mathrm{pc}$ after deconvolution with the disk (Dumke et al. 1997) and the $\lambda 850 \mu \mathrm{m}$ exponential scale height is only $110 \mathrm{pc}$ to the limits of those data. An equally significant result is that such high latitude gas is seen in a galaxy with a low SFR. To our knowledge, this is the first evidence for high latitude PAH emission in any external galaxy. A $\lambda 3.3 \mu \mathrm{m}$ PAH spur of $z$ extent $<120 \mathrm{pc}$ has now been detected by Tacconi-Garman et al. (2005) in the outflowing wind of the starburst galaxy, NGC 253, lending support to the concept of "mass-loaded" nuclear starburst winds and the possibility that some PAHs can survive such blasts, the intense UV radiation and shocks that are expected to accompany them.

The situation is quite different in NGC 5907, however, which does not show strong starbursting or outflowing winds. There is, however, a feature with anomalous velocities in high resolution $\mathrm{CO}$ data that cannot be explained by a bar or rotation model (Garcia-Burillo et al. 1997, their Feature F). A CO loop-like feature of size $\sim 20^{\prime \prime}$ associated with the anomalous structure in the CO maps is suggestive of a small-scale wind outflow. The radio point-source described in Sect. 4.4 (not at the same location as the CO feature) indicates that some SF activity is indeed occurring now in NGC 5907, although at a low level in comparison to other SF galaxies. The main independent evidence for high latitude emission in NGC 5907 is from the radio continuum (Dumke et al. 2000 and Fig. 14) which shows a $\lambda 20 \mathrm{~cm}$ thick disk scale length of $1.5 \mathrm{kpc}$ and a large scale field of $\sim 1 \mu \mathrm{G}$. This indicates that cosmic rays and magnetic fields do exist in the halo, although a detailed search for a correlation has not yet been possible (Sect. 4.4). 
A mechanism for depositing PAHs in the halo of a low SFR galaxy is not yet clear. Since SF and magnetic fields are both clearly present in NGC 5907, the PAHs may be vented into the halo initially from $\mathrm{SNe}$ and stellar winds from SF regions and then assisted in reaching high latitudes via coupling to the magnetic field. Excitation could be from UV photons that leak from the disk into the halo. An interesting possibility is that the 3-D VSGs are themselves converted into planar PAH macromolecules (Duley et al 2000). This would occur in regions of shocks and high UV excitation in the disk. The smaller PAHs may then be more easily "levitated" above the plane. Jones et al. (1996) have also noted that PAHs and smaller particles can build up at the expense of larger ones in shocks or winds and that wind velocities as low as tens of $\mathrm{km} \mathrm{s}^{-1}$ can modify the grain properties. The apparent dearth of $\lambda 850 \mu \mathrm{m}$ emission in the halo (Sect. 4.2) supports this view. It would be interesting to obtain more sensitive $\lambda 850 \mu \mathrm{m}$ and $\lambda 1.2 \mathrm{~mm}$ data in order to compare the results with starburst galaxies, such as NGC 5775 in which $\lambda 850 \mu \mathrm{m}$ emission has been detected in the halo (Brar et al. 2003). Determining the admixture of different dust components as a function of height would provide important clues as to what mechanisms may be involved. Conditions in galaxy halos are quite different from those in the disk and may more closely resemble those seen in elliptical galaxies.

Although there is a large optical ring around NGC 5907, as described in Sect. 1.1, there is no evidence that the high latitude PAH emission is directly related to it. The intersection of the optical ring with the disk of NGC 5907 occurs at two locations, one of which (to the north) is not in the field of view of our maps and the other is near the southern tip of the major axis (coordinates given in Fig. 11). We see no evidence for enhanced emission or extensions at the latter location. There does appear to be a disturbance in the $\mathrm{H} \alpha$ map of Rand et al. (1996) approximately at this location, however.

\section{Conclusions}

We have mapped the MIR emission of the low SFR galaxy, NGC 5907 , in the $6.7 \mathrm{~W}, 6.8 \mathrm{~N}, 7.7 \mathrm{~N}, 9.6 \mathrm{~N}, 11.3 \mathrm{~N}$, and $12 \mathrm{~W}$ ( $L W 2, L W 5, L W 6, L W 7, L W 8$, and $L W 10$, respectively) ISO bands, at high sensitivity and high resolution. Although we do not have spectra for NGC 5907, we have compared the line strengths and line ratios with those of other galaxies, especially M 83 in quiescent regions between the spiral arms. These comparisons indicate that the excitation conditions are quite typical of regions and/or galaxies with a low SFR. The contribution from hot VSGs is likely no more than $\sim 10 \%$ across these bands and is likely negligible in all bands except the broad, IRASequivalent $12 \mathrm{~W}$ band. The MIR emission is dominated by PAH emission.

In the disk, the high spatial resolution (1") broad band $12 \mathrm{~W}$ emission is peaked towards the optical dust lane. The bands dominated by PAH emission (at 6 " resolution) are observed all along the major axis of the galaxy and extend almost to the end of the optical disk (cf. $\sim 35 \mathrm{kpc}$ in comparison to $41 \mathrm{kpc}$ ) and possibly farther. The distribution of PAHs in the disk roughly follows that of the $\mathrm{CO}$ and not the broad HI distribution. The PAH distribution shows an apparent correlation with the SCUBA $\lambda 850 \mu \mathrm{m}$ distribution to within the positional errors of the data. The $\lambda 1.2 \mathrm{~mm}$ emission also follows that of the molecular gas. At the same time, there is some PAH emission (and also $\lambda 1.2 \mathrm{~mm}$ emission) at larger galactocentric radii than the $\mathrm{CO}$, suggesting that PAH emission is more widespread than the molecular gas alone. It is likely that greater PAH emission is seen where there is more molecular gas because there are more UV photons available from SF in these regions and because of the greater concentration of grains and PAHs in higher density gas. A smaller fraction of the PAH emission will be coming from the more extended HI, excited by the general diffuse ISRF.

An important finding of this work is the detection of PAH emission at high latitudes. The emission features show structure in the narrow bands that isolate single PAH features, appearing similar to features seen in $\mathrm{H} \alpha$, radio continuum, and other bands in other edge-on galaxies (see, e.g. Lee et al. 2002). Individual features extend to latitudes of up to $6.5 \mathrm{kpc}$ but a more typical scale height is $3.5 \mathrm{kpc}$. Previous high latitude emission on kpc scales has been seen only in the radio continuum component in this galaxy. Thus, cosmic rays and magnetic fields are known to exist in the halo, but other components have not (yet) been observed. Indeed, the PAHs appear to be selectively represented in the halo in comparison to large grains. We note also an increase in the $\lambda 11.3 / \lambda 7.7 \mathrm{PAH}$ ratio with distance from the major axis. Although NGC 5907 is a low SFR galaxy, there is some SF activity occurring, such as has been seen via some anomalous $\mathrm{CO}$ emission as well as a previously unreported off-nucleus radio continuum point source which is likely a supernova remnant or remnants. Thus, the halo PAHs may be transported to high latitudes with the help of coupling to magnetic fields (likely with gas as well), from regions of SF.

Although NGC 5907 is surrounded by a faint stellar ring, there is no indication in these data that interaction with the ring plays a direct role in the presence of the high latitude emission.

Acknowledgements. We are very grateful to Pierre Chanial for developing, at short notice, a seamless data reduction package for beamswitched observations. Many thanks also to Frédéric Galliano for his assistance with the process of ISO data reduction. Thanks to Elias Brinks for making the HI cube of this galaxy available for our use and to Manoulis Xilouris for providing the SCUBA $850 \mu \mathrm{m}$ data. We wish to thank Hervé Aussel also for providing software and assisting with checking the quality of the data and to Matthew Ashby for helpful suggestions. J.A.I. wishes to thank the director, administration and staff of CEA/Saclay for hospitality during a sabbatical leave. This work has been supported by the Natural Sciences and Engineering Research Council of Canada. This work was supported by the French National Programme: Physique Chimie du Milieu Interstellaire (PCMI).

\section{References}

Allamandola, L. J., Tielens, G. G. M., \& Barker, J. R. 1989, ApJS, 71, 733

Alton, P. B., Bianchi, S., Rand, R. J., et al. 1998, ApJ, 507, L125

Alton, P. B., Davies, J. I., \& Bianchi, S. 1999, A\&A, 343, 51

Alton, P. B., Xilouris, E. M., Misiriotis, A., Dasyra, K. M., \& Dumke, M. 2004, A\&A, 425, 109

Athey, A., Bregman, J., Bregman, J., Temi, P., \& Sauvage, M. 2002, ApJ, 571, 272 
Bendo, G. J., Joseph, R. D., \& Wells, M. 2002, AJ, 123, 3067

Bendo, G. J., Joseph, R. D., \& Wells, M. 2003, AJ, 125, 2361

Biviano, A., et al. 1998, The ISOCAM Dark Current Calibration Report, Technical Rep., ESA/ISO Data Centre

Bizyaev, D., \& Mitronova, S. 2002, A\&A, 389, 795

Blommaert, J., Siebenmorgen, R., Coulais, A. et al. 2003, CAM - The ISO Camera, in The ISO Handbook, Vol. II, see on-line version at http://www.iso.vilspa.esa.es/users/handbook/

Boulanger, F., Boissel, P., Cesarsky, D., \& Ryter, C. 1998, A\&A, 339, 194

Brar, R. 2005, Ph.D. Thesis, Queen's University

Brar, R., Irwin, J. A., \& Saikia, D. J. 2003, MNRAS, 340, 269

Cesarsky, C. J., Abergel, A., Agnese, P., et al. 1996, A\&A, 315, L32

Genzel, R., \& Cesarsky, C. J. 2000, ARA\&A, 38, 761

Chan, K.-W., Roellig, T. L., Onaka, T., et al. 2001, ApJ, 546, 273

Chanial, P. 2003, Ph.D. Thesis

Cohen, M., Allamandola, L., Tielens, A. G. G. M., et al. 1986, ApJ, 302,737

Condon, J. J. Cotton, W. D., Greisen, E. W., et al. 1998, AJ, 115, 1693

Coulais, A., \& Abergel, A. 2000, A\&AS, 141, 533

Dale, D. A. Silbermann, N. A., Helou, G., et al. 2000, AJ, 120, 583

Dale, D. A., Helou, G., Contursi, A., Silbermann, N. A., \& Kolhatkar, S. 2001, ApJ, 549, 215

Dekel, A., \& Silk, J. 1986, ApJ, 303, 39

Désert, F.-X., Boulanger, F., \& Puget, J. L. 1990, A\&A, 237, 215

Duley, W. W. 2000, ApJ, 528, 841

Dumke, M., Braine, J., Krause, M., et al. 1997, A\&A, 325, 124

Dumke, M., Krause, M., \& Wielebinski, R. 2000, A\&A, 355, 512

Galliano, F., Madden, S. C., Jones, A. P., et al. 2003, A\&A, 407, 159

Galliano, F. 2004, Ph.D. Thesis, l'Université de Paris XI Orsay

Garcia, A. M. 1993, A\&AS, 100, 47

Garcia-Burillo, S., \& Guelin, M. 1995, A\&A, 299, 657

Garcia-Burillo, S., Guelin, M., \& Neininger, N. 1997, A\&A, 319, 450

Groves, B. A., Cho, J., Dopita, M., \& Lazarian, A. 2003, PASA, 20, 252

Haas, M., Klaas, U., \& Bianchi, S. 2002, A\&A, 385, L23

Helou, G. 1986, ApJ, 311, L33

Helou, G., Malhotra, S., Hollenback, D., et al. 2001, ApJ, 548, L37

Howk, J. C., \& Savage, B. D. 1999, AJ, 117, 2077

Ichikawa, T., van Driel, W., Aoki, T., et al. 1994, ApJ, 433, 645

Irwin, J. A., English, J., \& Sorathia, B. 1999, AJ, 117, 2102

Irwin, J. A., Saikia, D. J., \& English, J. 2000, AJ, 119, 1592

James, P. A., \& Casali, M. M. 1998, MNRAS, 301, 280

Jarrett, T. H., Chester, T., Cutri, R., Schneider, S., \& Huchra, J. P. 2003, AJ, 125, 525

Johnston, K. V., Sackett, P. D., \& Bullock, J. S. 2001, ApJ, 557, 137

Jones, A. P., Tielens, A. G. G. M., \& Hollenbach, D. J. 1996, ApJ, 469,740

Kay, S. T., Pearce, F. R., Frenk, C. S., \& Jenkins, A. 2002, MNRAS, 330,113

Kennicutt, R. C., Jr. 1998, ApJ, 498, 541

Kessler, M. F., Steinz, J. A., Anderegg, M. E., et al. 1996, A\&A, 315, 27

Laurent, O. Mirabel, I. F., Charmandaris, V., et al. 2000, A\&A, 359, 887

Lee, S.-W., Irwin, J. A., Dettmar, R.-J., et al. 2001, A\&A, 377, 759

Lee, S.-W., Seaquist, E. R., Leon, S., Garcia-Burillo, S., \& Irwin, J. A. 2002, ApJ, 573, L107

Leger, A., \& Puget, J. L. 1984, A\&A, 137, L5

Lequeux, J., Fort, B., Dantel-Fort, M., Cuillandre, J.-C., \& Mellier, Y. 1996, A\&A, 312, L1

Lu, N. Helou, G., Werner, M. W., et al. 2003, ApJ, 588, 199

Misiriotis, A., Popescu, C. C., Tuffs, R., \& Kylafis, N. D. 2001, A\&A, 372,775
Marri, S., \& White, S. D. M. 2003, MNRAS, 345, 561

Mattila, K., Lemke, D., Haikala, L. K., et al. 1996, A\&A, 315, L353

Mattila, K., Lehtinen, K., \& Lemke, D. 1999, A\&A, 342, 643

Mo, H. J., \& Mao, S. 2004, MNRAS, 353, 829

Monaco, P. 2004, MNRAS, 352, 181

Morrison, H. L., Boroson, T. A., \& Harding, P. 1999, AJ, 108, 1191

Navarro, J. F., \& White, S. D. M. 1993, MNRAS, 265, 271

Neininger, N., \& Dumke, M. 1999, Proc. Natl. Acad. Sci., 96, 5360

Ohyama, Y. Taniguchi, Y., Iye, M., et al. 2002, PASJ, 54, 891

Peeters, E., Hony, S., Van Kerckhoven, C., et al. 2002, A\&A, 390, 1089

Peeters, E., Spoon, W. H. H., \& Tielens, A. G. G. N. 2004, ApJ, 613, 986

Popescu, C. C., Misiriotis, A., Kylafis, N. D., Tuffs, R. J., \& Fischera, J. 2000, A\&A, 362, 138

Popescu, C. C., Tuffs, R. J., Kylafis, N. D., \& Madore, B. F. 2004, A\&A, 414, 45

Rand, R. J. 1996, ApJ, 462, 712

Rengelink, R. B.,Tang, Y., de Bruyn, A. G., et al. 1997, A\&AS, 124, 259

Rigopoulou, D., Spoon, H. W. W., Genzel, R., et al. 1999, AJ, 118, 2625

Rossa, J., \& Dettmar, R.-J. 2003, A\&A, 406, 493

Roussel, H. Vigroux, L., Bosma, A., et al. 2001a, A\&A, 369, 473

Roussel, H. Sauvage, M., Vigroux, L., et al. 2001b, A\&A, 372, 406

Roussel, H., Sauvage, M., Vigroux, L., \& Bosma, A. 2001c, A\&A, 372,427

Rudy, R. J., Woodward, C. E., Hodge, T., Fairfield, S. W., Harker, D. E. 1997, Nature, 387, 159

Sackett, P. D., Morrison, H. L., Harding, P., \& Boroson, T. A. 1994, Nature, 370, 441

Sancisi, R. 1976, A\&A, 53, 159

Sanders, D. B., Mazzarella, J. M., Kim, D.-C., Surface, J. C., \& Soifer, B. T. 2003, AJ, 126,1607

Sasaki, T. 1987, PASJ, 39, 849

Scannapieco, E., Ferrara, A., \& Madau, P. 2002, ApJ, 574, 590

Sellgren, K. Luan, L, \& Werner, M. W. 1990, ApJ, 359, 384

Shang, Z., Brinks, E., Zheng, Z., et al. 1998, ApJ, 504, L23

Siebenmorgen, R., Blommaert, J., Sauvage, M., \& Starck, J.-L. 2000, The ISO Handbook III. CAM - The ISO Camera, Technical Rep., ESA/ISO Data Centre.

Sofue, Y. 1987, PASJ, 39, 547

Sofue, Y. 1994, PASJ, 46, 173

Soifer, B. T., Boehmer, L., Neugebauer, G., \& Sanders, D. B. 1989, AJ, 98, 766

Sommer-Larsen, J., Götz, M., \& Portinari, L. 2003, ApJ, 596, 47

Spekkens, K., Irwin, J. A., \& Saikia, D. J. 2004, MNRAS, 352, 1145

Spoon, H. W. W., Moorwood, A. F. M., Lutz, D., et al. 2004, A\&A, 414,873

Starck, J.-L., et al. 1999, A\&AS, 134, 135

Strickland, D. K. Heckman, T. M., Colbert, E. J. M., Hoopes, C. G., \& Weaver, K. A. 2004a, ApJS, 151, 193

Strickland, D. K. Heckman, T. M., Colbert, E. J. M., Hoopes, C. G., \& Weaver, K. A. 2004b, ApJ, 606, 829

Sturm, E., Lutz, D., Tran, D., et al. 2000, A\&A, 358, 481

Sturm, E., Lutz, D., Verma, A., et al. 2002, A\&A, 393, 821

Tacconi-Garman, L. E., Sturm, E., Lehnert, M., et al. 2005, A\&A, 432, 91

Thim, F., Tammann, G. A., Saha, A., et al. 2003, ApJ, 590, 256

Thompson, T. W. J., Howk, J. C., Savage, B. D. 2004, AJ, 128, 662

Tran, D. Q., Lutz, D., Genzel, R., et al. 2001, ApJ, 552, 527

Tsai, J. C., \& Mathews, W. G. 1995, ApJ, 448, 84 
Tuffs, R. J., Popescu, C. C., Völk, H. J., Madore, B. F., \& Gil de Paz, A. 2005, in The Dusty and Molecular Universe: a Prelude to Herschel and ALMA, ed. A. Wilson, ESA SP-577, 333

Uchida, K. I., Sellgren, K., Werner, M. W., \& Houdashelt, M. L. 2000, ApJ, 530, 817

Ulvestad, J. S., \& Ho, L. 2002, ApJ, 581, 925

Vermeij, R., Peeters, E., Tielens, A. G. G., \& van der Hulst, M. 2002, A\&A, 382, 1042

Veilleux, S., Shopbell, P. L., Rupke, D. S., Bland-Hawthorn, J., \& Cecil, G. 2003, AJ, 126, 2185

Verstraete, L., Puget, J. L., Falgarone, E., et al. 1996, A\&A, 315, L337
Vogler, A., Madden, S. C., Beck, R., et al. 2005, A\&A, 441, 491

Wang, Q. D., Chaves, T. A., \& Irwin, J. A. 2003, ApJ, 598, 969

Xilouris, E. M., Madden, S. C., Galliano, F., Vigroux, L., \& Sauvage, M. 2004, A\&A, 416, 41

Yost, S., et al. 1999, ApJ, 535, 644

Zepf, S. E., Liu, M. C., Marleau, F. R., Sackett, P. D., \& Graham, J. R. 2000, AJ, 119, 1701

Zhao, D. H., Jing, Y. P., Mo, H. J., \& Börner, G. 2003a, ApJ, 597, L9

Zhao, D. H., Mo, H. J., Jing, Y. P., \& Börner, G. 2003b, MNRAS, 339, 12

Zheng, Z. Shang, Z., Su, H., et al. 1999, AJ, 117, 2757 